\title{
Precise Inference of Expressive Units of Measurement Types
}

\author{
TONGTONG XIANG, University of Waterloo, Canada \\ JEFF Y. LUO, University of Waterloo, Canada \\ WERNER DIETL, University of Waterloo, Canada
}

\begin{abstract}
Ensuring computations are unit-wise consistent is an important task in software development. Numeric computations are usually performed with primitive types instead of abstract data types, which results in very weak static guarantees about correct usage and conversion of units. This paper presents PUnits, a pluggable type system for expressive units of measurement types and a precise, whole-program inference approach for these types. PUnits can be used in three modes: (1) modularly check the correctness of a program, (2) ensure a possible unit typing exists, and (3) annotate a program with units. Annotation mode allows human inspection and is essential since having a valid typing does not guarantee that the inferred specification expresses design intent. PUnits is the first units type system with this capability. Compared to prior work, PUnits strikes a novel balance between expressiveness, inference complexity, and annotation effort. We implement PUnits for Java and evaluate it by specifying the correct usage of frequently used JDK methods. We analyze 234k lines of code from eight open-source scientific computing projects with PUnits. We compare PUnits against an encapsulation-based units API (the javax.measure package) and discovered unit errors that the API failed to find. PUnits infers 90 scientific units for five of the projects and generates well-specified applications. The experiments show that PUnits is an effective, sound, and scalable alternative to using encapsulation-based units APIs, enabling Java developers to reap the performance benefits of using primitive types instead of abstract data types for unit-wise consistent scientific computations.
\end{abstract}

CCS Concepts: • Software and its engineering $\rightarrow$ Data types and structures; Empirical software validation; Software reliability; • Theory of computation $\rightarrow$ Program analysis; Program semantics; Type theory.

Additional Key Words and Phrases: Pluggable type system, Type inference, Units of measurements, Dimensional analysis, Scientific computing

\section{ACM Reference Format:}

Tongtong Xiang, Jeff Y. Luo, and Werner Dietl. 2020. Precise Inference of Expressive Units of Measurement Types. Proc. ACM Program. Lang. 4, OOPSLA, Article 142 (November 2020), 28 pages. https://doi.org/10.1145/ 3428210

\section{INTRODUCTION}

Ensuring computations are unit-wise consistent is an important task in software development for scientific, engineering, and business domains. Performing calculations with mismatched dimensions (e.g., length, time, speed, etc.) or units (e.g., meters, millimeters, seconds, meters-per-second, etc.) can result in catastrophic failures. For efficiency reasons, such computations are usually performed with primitive types instead of abstract data types, which results in very weak static guarantees about correct usage and conversion of units.

Authors' addresses: Tongtong Xiang, Electrical and Computer Engineering, University of Waterloo, Canada, txiang@ uwaterloo.ca; Jeff Y. Luo, Electrical and Computer Engineering, University of Waterloo, Canada, j36luo@uwaterloo.ca; Werner Dietl, Electrical and Computer Engineering, University of Waterloo, Canada, wdietl@uwaterloo.ca.

This work is licensed under a Creative Commons Attribution 4.0 International License.

(c) 2020 Copyright held by the owner/author(s).

2475-1421/2020/11-ART142

https://doi.org/10.1145/3428210

Proc. ACM Program. Lang., Vol. 4, No. OOPSLA, Article 142. Publication date: November 2020. 


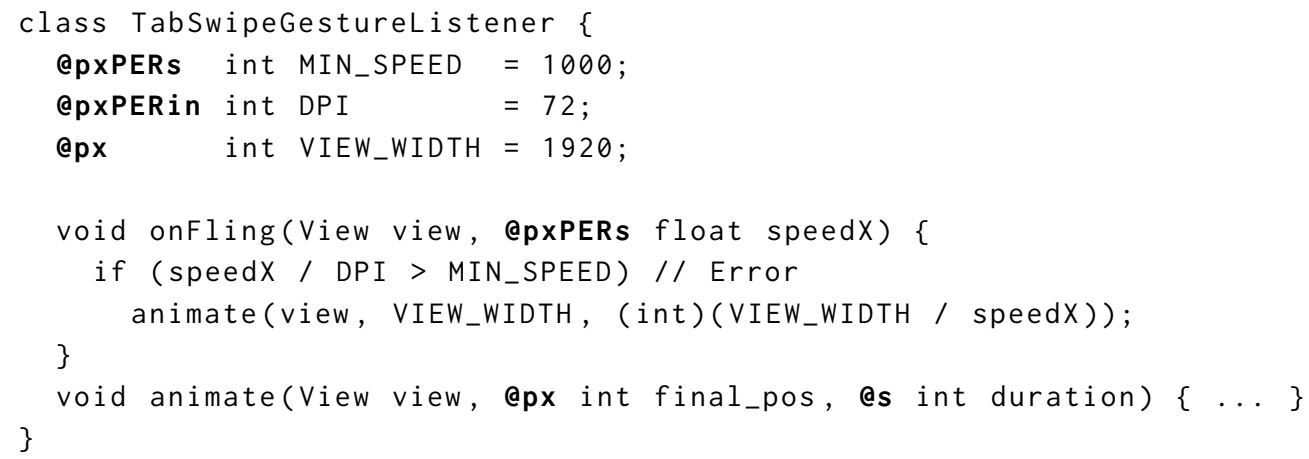

Fig. 1. Minimized and annotated example of the Firefox Android swipe-to-close error. The type qualifiers @px, @s, @pxPERs, and @pxPERin represent pixels, seconds, pixels-per-second, and pixels-per-inch, respectively. Type qualifiers are implemented as Java 8 type annotations [Ernst et al. 2012]. These qualifiers are not present in the original code [Johnston 2012]. For the annotated code, PUnits produces an error message for the comparison on line 7 .

The Mars Climate Orbiter disintegrated in 1998 on its approach to Mars due to one software component communicating values in Imperial units, while the receiving component expected values in International System (SI) units [Board 1999]. This mismatch cost US taxpayers $\$ 327.6$ million USD.

In 2012, Mozilla added a swipe-to-close feature to its Firefox browser for Android. An error in the implementation prevented users from activating this feature. The relevant code is presented in Fig. 1. The initial minimum swipe speed was set to 1000 pixels-per-second and defined as an integer constant MIN_SPEED in the program (line 2). If a user swipes a browser tab with a speed (speedX) exceeding this minimum, the code would animate the closing of the tab (line 8). Subsequently, the feature was updated to adapt to different devices by performing the computations with respect to the screen resolution in pixels-per-inch (line 7). A bug report [Johnston 2012] indicates that the swipe speed was not modified during the feature update. As a result, the integer was interpreted as 1000 inches-per-second, effectively disabling the feature.

As another example, two errors in Daikon[Ernst et al. 2007], a dynamic likely invariant detection tool, were caused by mismatches between seconds and milliseconds[Daikon 2003, 2004]. The standard Java type system does not prevent such errors, as the computations are performed on primitive values.

This paper presents PUnits, an expressive type system to enforce units of measurement and a precise whole-program type inference approach that helps developers annotate code with unit types. PUnits is implemented for Java as an optional type system [Bracha 2004]. It handles all of Java's language features and works on real-world Java applications. Nevertheless, the idea for PUnits is not limited to Java and can be widely adopted.

PUnits can detect the error in the Firefox example with the annotated version of the code shown in Fig. 1. The type qualifiers specify the units of the program elements, and PUnits reasons about the correctness of the code by checking their qualified types. Developers utilize PUnits by adding annotations, type checking their program, and then fixing errors or adding additional annotations PUnits reports an error as it computes the resulting unit of the division on line 7 to be ainPERs (inches-per-second), which is not comparable with @pxPERs (pixels-per-second). There are multiple ways to resolve this error, by converting the left hand side to @pxPERs, converting the right hand 
side to @inPERs, or not comparing these at all. As PUnits cannot interpret the developer's intent on whether an operation is meant as a unit conversion or as an arithmetic computation, the developer has to be explicit when converting between units. For example, if the developer wants to convert pixels @px to inches @in, they can either specify a variable annotated with @pxPERin and divide the @px by @pxPERin to get the @in, or they can invoke a unit conversion method that takes in a parameter in @px and returns a value in @in. This enables PUnits to enforce correct unit conversions. For Daikon, PUnits detects reproductions of the units errors. We fully annotated the Daikon source code and PUnits guarantees that these kinds of errors will not be introduced again.

Developers can also use PUnits to perform whole-program type inference to check whether there exists any valid typing. With only animate() fully annotated, PUnits infers speedX to be @pxPERs. The choice of the unit for DPI determines the unit for MIN_SPEED and vice versa. PUnits determines that the program has at least one valid typing. It can infer one variable to be dimensionless and the other to @pxPERs. The programmer inspects the inference results for the two variables and realizes that the solution does not match their design intent, which indicates there is an error. The programmer then annotates DPI and MIN_SPEED with the intended units. PUnits then concludes that there is no valid typing and produces an error on line 7. Note how this error would have been missed without a human looking at the inference results and deciding whether the result matches their expectations. Therefore, PUnits also provides whole-program type inference with annotation mode, where the most precise units are inserted back into the source code to allow human inspection and to provide well-specified applications and libraries. PUnits is the first units type system with this capability.

PUnits makes contributions to the expressiveness, precision, usability, effectiveness, and soundness of units of measurement types. The system, benchmarks, and the formalization are openly available. $^{1}$

- PUnits is expressive: it is parameterized by the set of base units (for example, the seven SI base units), and can represent all units defined as products and quotients of the base units. The design is not limited to SI units; a developer can provide base units for any custom measurement system such as pixels, bytes, and currencies. The system is also parameterized by the set of mathematical operations, each supported by a function which gives the resulting unit of applying the mathematical operation to two units. PUnits can be instantiated for dimensional analysis by supplying a set of base dimensions instead of base units.

- PUnits is precise: our whole-program type inference approach infers the most precise typing possible when more than one type-safe solution is permitted. When inserted into source code, a precise solution prevents unintended new uses of well-annotated code. To the best of our knowledge, no prior work has attempted to infer units which are inserted into the source code; they are traditionally only used to determine whether the program type checks.

- PUnits is easy to use: we implemented defaulting rules, method-local flow-sensitive type refinement, parametric polymorphism over units, and receiver-dependent types to minimize the need for unit annotations in method bodies, removing unnecessary clutter. No other units type systems have receiver dependent types. By adding a few key unit specifications in application code or in commonly used libraries, inference can automatically discover and annotate every relevant program element with type-safe unit specifications.

- PUnits is effective: we present case studies of using PUnits for eight Java projects, including Daikon, totalling 234k lines of code. PUnits is evaluated against an encapsulation-based units API and the experiments show PUnits can discover and prevent units-related errors in real-world projects, which an encapsulation-based units API has failed to detect. PUnits

$\overline{{ }^{1} \text { https://opprop.github.io }}$ 
inferred 90 scientific units for five Java projects, and created well-specified applications and libraries with low annotation effort. PUnits reaps the performance benefits of using primitive types instead of abstract data types to achieve unit-consistent programs.

- PUnits is sound: we proved a minimal formalization of the system sound in Coq [Coq Development Team 2004].

Compared to prior work, PUnits strikes a novel balance between expressiveness, inference complexity, and annotation effort. PUnits is a practical tool for improving the quality of software and can help developers write unit-correct code. PUnits builds on techniques from type qualifier systems and constraint-based type inference, expanding them to the first system that provides a practical unit type system. Our implementation and evaluation of PUnits shows that these techniques are necessary and are effective in ensuring the unit correctness of real-world programs. Our approach and implementation can be used to define other pluggable type systems. The type rules only need to be defined once and our framework provides all three usage modes.

This paper is organized as follows. Sec. 2 presents an overview of PUnits and its type checking and inference approach. Sec. 3 presents the formalization and summarizes the Coq proof. Sec. 4 presents the design and implementation details of PUnits. Sec. 5 presents the case studies. Sec. 6 compares PUnits to related work. Finally, Sec. 7 concludes and discusses possible future work.

\section{OVERVIEW}

A type system for units of measurements must be expressive enough to allow the use of different measurement systems. The system must be robust to changes in the supported units and enable reusable code with selective sensitivity to units. The system must also be able to efficiently reason about program correctness, independent of which particular measurement systems are used. Finally, the system must support developers in terms of their workflows and minimize the burden required to use the system.

A pluggable type system [Bracha 2004] enhances the type system of a language to enforce stronger semantics and provide additional static guarantees. Pluggable type systems provide a set of type qualifiers to imbue additional semantic meaning to the types of a language, and enforce custom semantics through a set of type rules expressed over qualified types. Developers can annotate their code with type qualifiers and optionally use a suite of pluggable type systems to check their programs for potential errors that the standard type system of a language does not prevent.

PUnits is a pluggable type system that allows developers to specify units of measurement systems by defining a set of base units, and to specify the units of their program elements via type qualifiers. Each type qualifier specifies a single unit. For example, @m int is a qualified type specifying an integer with the unit of meters. The qualifier @Dimensionless specifies the unit of truly dimensionless quantities, such as number literals, $e$, and $\pi$, and usually does not need to be written. The qualifiers are organized as a flat lattice over the set of scientific units, with top $T$ and bottom $\perp$ type qualifiers. T expresses unit-wise agnostic types. To support object-oriented languages, $\perp$ is used for null types, and $T$ and $\perp$ are respectively used as the upper and lower bounds of generic type parameters. We use the term unit to denote both the scientific unit of a program element as well as the type qualifier used to annotate the program element.

Developers provide partially-annotated source code and specifications for library code as input to PUnits, which first normalizes the units into an internal representation (Sec. 2.1). PUnits enforces unit-consistent computations by generating and solving typing constraints through syntax-guided constraint generation rules. A user of PUnits can pick among one of three modes: 1) modular type checking (Sec. 2.2), 2) whole-program type inference to ensure valid typing (Sec. 2.3), and 3) wholeprogram type inference to annotate the program with the most precise set of units (Sec. 2.3.1). The 


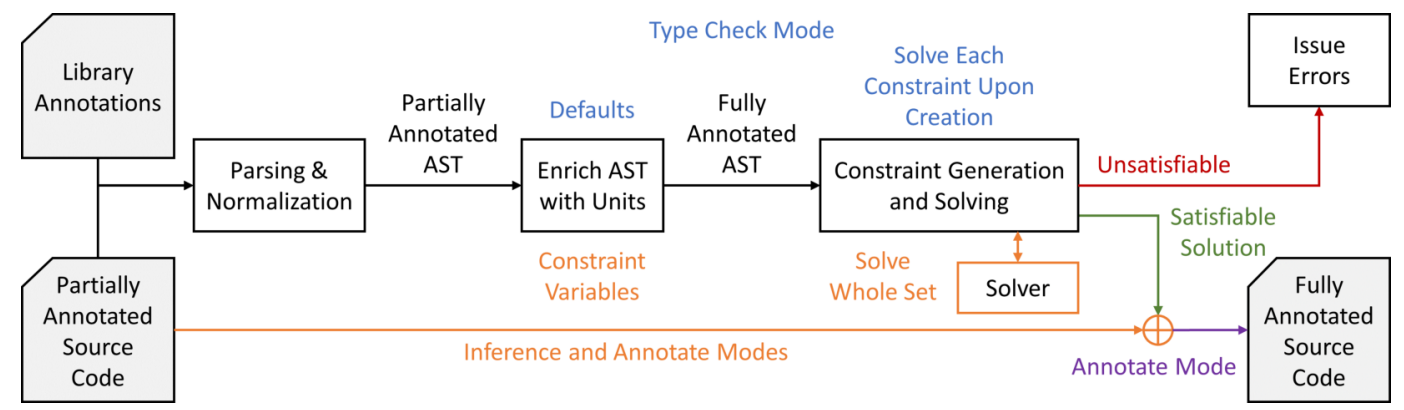

Fig. 2. Type checking and inference process with three different modes: 1) Modular type checking using defaults and eager constraint solving; 2) Whole-program type inference to ensure valid typing; and 3) Wholeprogram type inference with additional breakable constraints to annotate code with the most precise set of units.

modes are respectively given the short names of type check mode, inference mode, and annotate mode. The type rules are defined once and then used in all three modes. Fig. 2 illustrates the overall type checking and type inference process of PUnits.

\subsection{Base Units and the Normalized Representation of Units}

PUnits internally represents all units through a normalized representation to efficiently reason about program correctness. A base unit is the unit of measurement from which all other units from the same dimension can be derived. Each base unit is represented by a symbol. For SI, the base units are $\left\{\mathrm{m}, \mathrm{s}, \mathrm{g}^{2}, \mathrm{~A}, \mathrm{~cd}, \mathrm{~K}, \mathrm{~mol}\right\}$.

$P U n i t s$ represents scientific units as a single prefix and a product of one or more base units, each raised to an integer power: $p \overline{u^{z}}$ (where $\bar{X}$ denotes a sequence of elements $X_{1}, \ldots, X_{k}$ ). Each base unit appears exactly once.

The representation can support any unit system. In the Java implementation, $p$ is encoded as a base-10 prefix with an integer exponent to support SI-like units: $p=10^{z}$. Thus, we need to declare additional base units for units that are not a base 10 multiple (e.g., inch vs. cm). Fig. 3 shows some examples of how units are represented in PUnits. The prefix $p$ could be encoded as floating-point value. However, the precision of analysis will be subjected to floating-point rounding errors, and safe floating-point comparisons must be used. The set of base units is customizable, allowing for base units such as currencies, abstract quantities, lengths, and pixels. There are special cases where the units alone cannot decide whether a computation is permitted. For example, a radian and a steradian, respectively defined as $\mathrm{m} / \mathrm{m}$ and $\mathrm{m}^{2} / \mathrm{m}^{2}$, would both be normalized to dimensionless. In PUnits, radian and steradian can be declared as two different base units. Type systems that only allow SI units cannot support such derived units. Some derived units have several different representations, such as energy, which can be kinetic, thermal, electrical, etc. These representations can be supported in PUnits by declaring distinct base units. The representation is compact, and allows for efficient calculation of the resulting units of various arithmetic operations. Two units are equal if and only if every component in their normalized representations are pairwise equal.

PUnits can also be instantiated for dimensional analysis by using a set of base dimensions (e.g. @Length) instead of base units (e.g. @m), together with the fixed prefix $p=1$. Dimensional analysis can be useful if the developer does not care about the specific units, such as verifying if two units

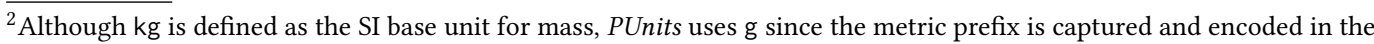
prefix component of the normalized representation.
} 


\begin{tabular}{|c|c|c|c|c|c|}
\hline Unit & $10^{z}$ & $\mathrm{~g}^{z}$ & $\mathrm{~m}^{z}$ & $\mathrm{~s}^{z}$ & $\ldots$ \\
\hline $\mathrm{m}^{2}$ & 0 & 0 & 2 & 0 & 0 \\
\hline $\mathrm{kg}$ & 3 & 1 & 0 & 0 & 0 \\
\hline $\mathrm{N}$ & 3 & 1 & 1 & -2 & 0 \\
\hline $\mathrm{kN}$ & 6 & 1 & 1 & -2 & 0 \\
\hline Dimensionless & 0 & 0 & 0 & 0 & 0 \\
\hline
\end{tabular}

Fig. 3. Example unit representations. Squared-meter is represented as $\mathrm{m}^{2}$ and kilogram is represented as $10^{3} \mathrm{~g}$ with $\mathrm{g}$ as the base unit. All SI prefixes of a unit are multiplied together into one prefix. Newton is represented by $10^{3} \mathrm{~g} \mathrm{~m} \mathrm{~s}^{-2}$ and a kilo-newton is normalized and represented by $10^{6} \mathrm{~g} \mathrm{~m} \mathrm{~s}^{-2}$. QDimensionless is represented by $10^{0}$.

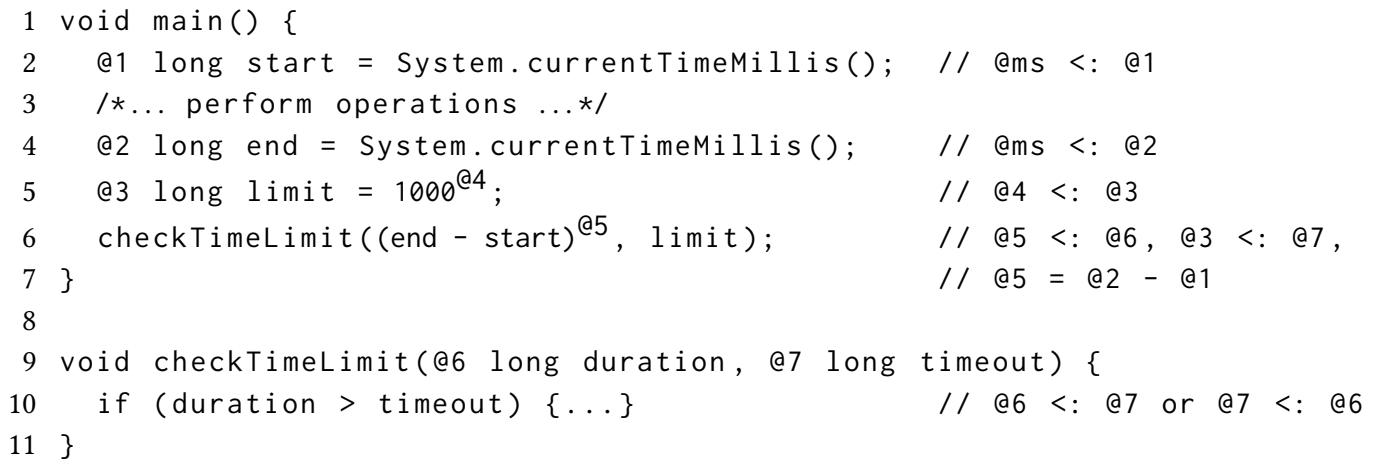

\begin{tabular}{|l|c|c|c|c|c|c|c|}
\hline Constraint Variable & @1 & @2 & @3 & @4 & @5 & @6 & @7 \\
\hline Type Check Mode & @ms & @ms & $\mathrm{D}$ & $\mathrm{D}$ & @ms & $\mathrm{D}$ & $\mathrm{D}$ \\
\hline Inference Mode & $\mathrm{T}$ & $\mathrm{T}$ & $\mathrm{T}$ & $\mathrm{T}$ & $\mathrm{T}$ & $\mathrm{T}$ & $\mathrm{T}$ \\
\hline Annotate Mode & @ms & @ms & @ms & @ms & @ms & @ms & @ms \\
\hline
\end{tabular}

Fig. 4. A program which performs a number of operations and then checks to see if the operations were performed within a time limit. Placeholders @1 to @7 are used to mark the locations for which types need to be determined. System. currentTimeMillis() is annotated to return values with the unit of ams (milliseconds). The table below the code presents the units assigned to the placeholders @1 through @7 in each of the three solving modes, where D stands for @Dimensionless. Only @4, @6, and @7 are inserted back into the source code.

are convertible. Unit-wise analysis offers more precision than dimensional analysis and is able to detect more errors.

\subsection{Modular Type Checking}

Modular type check mode allows methods and classes to be type-checked independently. It gives developers quick feedback on potential units-related errors, with a method-local view of potential problems.

PUnits uses defaults for missing units (Sec. 4.1.1). The defaults are chosen to give errors across method and class boundaries, allowing developers to quickly catch potential problems and focus on fixing one method or class at a time. A set of mandatory constraints (Sec. 3.2) is generated by 
syntax-guided constraint generation rules (Sec. 3.3). In type check mode, each constraint is solved upon creation. Unsatisfied constraints produce compilation errors.

The modes are illustrated through the example presented in Fig. 4, together with the constraints generated for each line. In type check mode, after applying defaults and method-local type refinement (Sec. 4.1.2), without adding any additional annotations, initial type checking will fail. An error is issued on line 6 for passing a @ms value to a parameter which expects a @Dimensionless value. Type check mode gives method-local views of potential errors, some of which may be due to a lack of annotations in the code. By iteratively applying the type check mode, all units mismatches in the code will be revealed. The method call and comparison are type-safe if @3, @4, @6, and @7 are annotated with @ms.

\subsection{Whole-Program Type Inference}

Whole-program type inference mode ensures a valid typing exists for a program and, optionally, infers the most precise units for a program (see Sec. 2.3.1 below). Inference mode pinpoints the set of code locations across the program that together could cause a units-related error.

In whole-program type inference, constraint variables are created, which are placeholders for concrete units. The same mandatory constraints (Sec. 3.2) are generated by syntax-guided constraint generation rules (Sec. 3.3), as in type check mode. The constraints for the whole program are collected and solved together by a MaxSMT solver (Sec. 4.3). Multiple type-safe solutions are possible in inference mode and, as long as a solution exists, inference succeeds.

In Fig. 4, the constraints are solved together in inference mode and determined to be satisfiable. One possible solution assigns $T$ as the unit for all constraint variables. The program is therefore type safe. However, this solution does not use the most precise units that could be assigned to the constraint variables.

As a simple unsatisfiable example, we can annotate parameter duration on line 9 with @s. From lines 2 and 4, the constraints require @1 and @2 to be @ms or $\mathrm{T}$. This propagates to the subtraction on line 6, and @5 must be @ms or T. Since neither unit is a subtype of @s, the constraints introduced in lines 2, 4, and 6 are together unsatisfiable.

If inference succeeds, there exists a valid typing for the program. If a typing exists, inference will succeed. However, type checking with default qualifiers can fail for different reasons than type inference. Errors given in inference mode provide a whole-program view of the reasons why no type-safe solutions can be inferred, and indicate potential errors.

2.3.1 Annotation Mode. Annotation mode is whole-program type inference with additional, breakable, preference constraints (Sec. 4.3). It annotates the program with the most precise units possible. We rely on the SMT solver to produce the optimal solution guided by hard and soft constraints provided to the solver. The annotation mode inserts the inferred solutions back into the source code, giving the developer a chance to inspect the results. This is intended to help developers create well-specified applications and libraries.

For example, if a parameter can be annotated with @m or $T$ as its unit, then @m is the more precise typing and is preferred over the more general typing $T$. The soft constraint added to the subtype constraint tells the solver to prefer the subtype (concrete units) instead of the supertype $T$.

PUnits chooses to infer the most precise typing to prevent accidental misuses of annotated code by newly added code. PUnits also prefers inferring @Dimensionless as a unit for under-constrained constraint variables. Preferring units to be @Dimensionless will raise errors when developers introduce additional units into a program. Annotate mode inserts all non-default units from its solution into the corresponding types in source code. Annotations on local variables are also not inserted, because flow-sensitive type refinement can infer such annotations (Sec. 4.1.2). This decreases noise 


$$
\begin{aligned}
& \text { Program } P::=\overline{v d} \bar{s} \\
& \text { Variable Declaration } v d::=T v=z \\
& \text { Assignment Statement } \quad s::=v=e \\
& \text { Expressions } e::=l|v| \text { e op e } \\
& \text { Labeled Literal } \quad l::=T z \\
& \text { Types } T::=\top|\perp| p \overline{u^{z}} \mid \alpha
\end{aligned}
$$$$
\text { Arithmetic Operations op }::=+|-| *|\div| \ldots
$$$$
\text { Base Units } u::=\ldots
$$
$v$ variable identifier
$p$ prefix number literal
$z$ signed integer literal
$\alpha$ constraint variable identifier

Fig. 5. Syntax and naming conventions. Constraint variables $\alpha$ are only used in inference and annotate modes, as placeholders for concrete units. The set of base units $u$ and the set of arithmetic operators op can be customized.

in the fully-annotated program. Like in inference mode, if any of the mandatory constraints cannot be satisfied, errors are raised for the code locations which generated the unsatisfiable constraints.

When using annotation mode for the example in Fig. 4, the solution assigns @ms to each constraint variable as it is the most precise unit for each of the types. The solution for @4, @6, and @7 are inserted into the source code. The other constraint variables are refined and do not need to be inserted. The developer can continue to type check and infer units for a larger program, ensuring all calls to checkTimeLimit() must pass @ms arguments. In this example, both ams and T would be possible inference solutions. By using the more precise @ms, the API expresses a concrete unit, instead of using the $T$ type.

\section{PUNITS TYPE SYSTEM AND FORMALIZATION}

This section formalizes the PUnits type system and inference approach and presents a core calculus, $\pi$ Units, as a minimal imperative language with integers, arithmetics, and units. We proved soundness in Coq [Coq Development Team 2004] and the proof scripts are openly available. ${ }^{3}$

\subsection{Syntax of $\pi$ Units}

The syntax of $\pi$ Units and the naming conventions are presented in Fig. 5. A program $P$ consists of a sequence of variable declarations $\overline{v d}$, followed by a sequence of assignment statements $\bar{s}$. A variable declaration $v d$ pairs a type $T$ with an identifier $v$, and assigns an initial integer value $z$ to the variable. An expression $e$ can be a labeled literal $l$, a read of a variable $v$, or an arithmetic operation $e$ op $e$ between two sub-expressions for some operation $o p$. A labeled literal is an integer literal $z$ labeled with a type $T$. The set of types $T$ consists of top $T$, bottom $\perp$, and normalized unit representations $p \overline{u^{z}}$ (see Sec. 2.1). In inference and annotate modes, $T$ also includes constraint variables $\alpha$.

\subsection{Constraint Variables and Typing Constraints}

A constraint variable $\alpha$ is a placeholder for a concrete unit. A fresh constraint variable is introduced for each location that is missing a unit and for the resulting unit of each arithmetic operation. Inference assigns one concrete unit to each constraint variable. $\pi$ Units generates three kinds of constraints $\sigma$ over types:

- Well-formedness constraint $w f(\alpha)$ : enforces that any satisfying solution for constraint variable $\alpha$ is uniquely interpretable as a single unit.

- Subtype constraint $T_{a}<: T_{b}$ : enforces $T_{a}$ to be the same type or a subtype of $T_{b}$.

\footnotetext{
${ }^{3}$ https://github.com/opprop/units-inference/tree/master/coq-proof
} 


$$
\begin{aligned}
& \begin{aligned}
\text { Program: } & \emptyset \vdash \overline{v d}: \Gamma, \Sigma_{\overline{v d}} \quad \Gamma \vdash \bar{s}: \Sigma_{\bar{s}} \\
\hline \vdash P: \Gamma, \Sigma & \vdash \overline{v d} \bar{s}: \Gamma, \Sigma_{\overline{v d}} \cup \Sigma_{\bar{s}} \\
\text { Variable Declaration: } & \frac{\vdash T \mathrm{OK}: \Sigma_{v d}}{\Gamma \vdash T v=z: \Gamma\{v \rightarrow T\}, \Sigma_{v d}} \\
\Gamma \vdash v d: \Gamma^{\prime}, \Sigma & \frac{\Gamma \vdash v: T_{v}, \emptyset \quad \Gamma \vdash e: T_{e}, \Sigma_{e} \quad \vdash T_{e}<: T_{v}: \Sigma_{<:}}{\Gamma \vdash v=e: \Sigma_{e} \cup \Sigma_{<:}} \\
\text {Statement: } & \frac{\Gamma \vdash s: \Sigma}{\Gamma \vdash s}
\end{aligned} \\
& \begin{array}{l}
\text { Expression: } \\
\Gamma \vdash e: T, \Sigma
\end{array} \overline{\Gamma \vdash T z: T, \emptyset} \frac{T=\Gamma(v)}{\Gamma \vdash v: T, \emptyset} \\
& \frac{\alpha \text { fresh } \Gamma \vdash e_{1}: T_{1}, \Sigma_{1} \quad \Gamma \vdash e_{2}: T_{2}, \Sigma_{2}}{\Gamma \vdash e_{1} \text { op } e_{2}: \alpha, \Sigma_{1} \cup \Sigma_{2} \cup\left\{w f(\alpha), \alpha=T_{1} \text { op } T_{2}\right\}}
\end{aligned}
$$

Fig. 6. Constraint generation rules. The sequence forms of the judgments for $\Gamma \vdash \overline{v d}: \Gamma^{\prime}, \Sigma$ and $\Gamma \vdash \bar{s}: \Sigma$ are standard and are presented in Fig. 20 in Appx. A. Helper judgments are defined in Fig. 21 in Appx. A. The generated constraint set $\Sigma$ encodes all constraints that need to be satisfied to give a type-correct program.

- Arithmetic constraint $\alpha=T_{a}$ op $T_{b}$ : enforces that $\alpha$ will be equal to the result of the arithmetic computation of $T_{a}$ op $T_{b}$, for some defined arithmetic operation op. $\pi$ Units abstracts over a concrete set of arithmetic operations. op constraints build upon the idea of Viewpoint Adaptation [Dietl et al. 2007, 2011b] and can be thought of as computing the result type of the arithmetic operator $o p$.

\subsection{Syntax-Directed Constraint Generation Rules}

Fig. 6 shows the syntax-directed constraint generation rules. An environment $\Gamma$ maps variables to their types. Notation $\Gamma(v)$ looks up the type of a variable $v$ from $\Gamma$. A constraint set $\Sigma$ contains the constraints $\sigma$ needed to satisfy a particular rule. A variable declaration requires that its declared type is well-formed, given by the well-formed judgment ( $\vdash T$ OK) defined in Fig. 21 in Appx. A. This judgment generates a well-formedness constraint for the constraint variables. All other types are always well-formed. A variable declaration updates the environment $\Gamma$ by mapping variable $v$ to its declared type $T$, and produces constraint $\Sigma_{v d}$. An assignment statement requires that the type of the expression be a subtype of the variable type. Helper subtype judgment $\left(-T_{e}<: T_{v}: \Sigma\right)$ defined in Fig. 21 in Appx. A produces a constraint if either the type of the variable or expression is a constraint variable. The constraints for an assignment includes this subtype constraint and any constraints from the expression.

The rules for expressions are as follows. For a labeled literal expression $T z$, the type of the value is returned. For a variable read expression $v$, the type of the variable is retrieved by look-up in $\Gamma$. The judgments for labeled literals and variable reads do not generate any constraints. For arithmetic expressions, $\pi$ Units generates a fresh constraint variable $\alpha$ to hold the result type of the operation, a well-formedness constraint for $\alpha$, an arithmetic constraint ( $\alpha=T_{1}$ op $T_{2}$ ), and the constraints generated for the sub-expressions.

\subsection{Small-Step Operational Semantics}

$\pi$ Units models a stack frame $F$ as a map of variables to their static types $T_{v}$ and their labeled values $T_{l} z$. The label type of a value $T_{l}$ is its declared type or a type computed at runtime. 


$\begin{array}{ll}\text { L-ST-Consistent: } & T_{1}^{\prime}<: T_{1} \Longrightarrow\left(T_{1}^{\prime} \text { op } T_{2}\right)<:\left(T_{1} \text { op } T_{2}\right) \\ \text { R-ST-Consistent: } & T_{2}^{\prime}<: T_{2} \Longrightarrow\left(T_{1} \text { op } T_{2}^{\prime}\right)<:\left(T_{1} \text { op } T_{2}\right)\end{array}$

Fig. 7. $o p$ and Subtype Consistent Axioms.

In $\pi$ Units, a program can evaluate to STUCK if a variable with type $T_{v}$ is assigned a value with type $T_{l}$ and $T_{l}$ is a supertype of $T_{v}$. This subtype requirement in the operational semantics models misusing computed results with mismatching units. A program does not get STUCK during arithmetic computations in $\pi$ Units. This is required for our soundness proof to show that well-typed programs never get stuck.

Aside from the frame model, the operational semantics is standard. The details are presented in Fig. 22 in Appx. A.

\subsection{Coq Proof Summary}

We prove soundness of type checking in $\pi$ Units using the standard approach of proving progress and preservation theorems [Wright and Felleisen 1994]. The proof omits modeling constraint variables $\alpha$, as they do not arise for type checking. The two theorems and additional supporting lemmas and summaries of their proofs are presented in Appx. B.

An interesting subcase arises for preservation during expression reductions. Lemma 3.1 defines the supporting lemma.

LEMMA 3.1 (PRESERVATION DURING EXPRESSION REDUCTION).

$$
\Gamma \vdash e: T \wedge \vdash F \mathrm{OK} \wedge F, e \Rightarrow e^{\prime} \Longrightarrow \exists T^{\prime} \cdot \vdash T^{\prime}<: T \wedge \Gamma \vdash e^{\prime}: T^{\prime} .
$$

The type of a value stored in a variable can be a subtype of the static type of the variable. For the subcase where $e=e_{1}$ op $e_{2}$ with static type $T_{1}$ op $T_{2}$ for some given operation $o p$, we must show that the run-time type of the expression is always a subtype of its static type. This then ensures that the type of the final result of the computation is always a subtype of the static type of the variable to which it is being assigned, thus the program never gets STUCK.

If $e_{1}$ can reduce to $e_{1}^{\prime}$, by the inductive hypothesis $\Gamma \vdash e_{1}^{\prime}: T_{1}^{\prime}$ for some $T_{1}^{\prime}<: T_{1}$ then $\Gamma \vdash e^{\prime}$ : $T_{1}^{\prime}$ op $T_{2}$. We are left with the obligation that $\left(T_{1}^{\prime}\right.$ op $\left.T_{2}\right)<:\left(T_{1}\right.$ op $\left.T_{2}\right)$, which is imposed upon the definition of $o p$ for any given $o p$. Similarly, if $e_{2}$ can be reduced to $e_{2}^{\prime}$ and $\Gamma \vdash e_{2}^{\prime}: T_{2}^{\prime}$, the obligation $\left(T_{1}\right.$ op $\left.T_{2}^{\prime}\right)<:\left(T_{1}\right.$ op $\left.T_{2}\right)$ is also imposed on the definition of op. The two obligations are defined as the L-ST-Consistent and R-ST-Consistent axioms shown in Fig. 7. While the op between scientific units must obey the arithmetic laws, such as $a^{n} \times a^{m}=a^{n+m}$, the axioms inform how to define op between $T$ or $\perp$ with a unit and with each other.

With operations defined in a way that satisfies these two axioms, the run-time type of the final result of reducing a well-typed expression will always be a subtype of the static type of the expression. Since the type rules require the type of an expression to be a subtype of a variable during an assignment, by subtype transitivity the run-time type of an expression is also a subtype of the variable. The proof concludes that the frame is always well-formed during execution and a well-typed $\pi$ Units program never gets STUCK.

Note that in $\pi$ Units, a program never gets stuck during arithmetic operations. For example, a valid definition of addition satisfying the axioms is the least-upper-bound, which permits $a m+@ k m$, giving $T$ as the result type. An error is only raised if the resulting value is passed to something that cares about the units. If the assignment context has a concrete unit or is dimensionless, the constraint will propagate back into the binary operation. $\pi$ Units permits computations if the result is not unit dependent. 


\subsection{Extending $\pi$ Units to the Full Java Language}

Although $\pi$ Units is minimal, it sufficiently models the necessary core calculus to support all of PUnits's features and extends to the full Java language.

$\pi$ Units models variable declarations, reads, and updates, which directly model Java fields and local variables. Wherever Java performs a subtype check, such as for method invocations, subclassing and method overloading, or generic type argument bounds, PUnits also performs a subtype check or encodes a subtype constraint.

$\pi$ Units also models arithmetic operations, which models the checks and constraints generated by PUnits for Java's arithmetic operations through the binary operators or through methods such as Math. addExact (int $\mathrm{x}$, int $\mathrm{y}$ ). PUnits provides definitions of addition, subtraction, multiplication, division, and modulo which satisfy the op and subtype consistency axioms. The encoding of the operations for the solver are presented in Sec. 4.3. Supporting a range of mathematical operations is a feature of $\pi$ Units. However, it is not expected of the developer to provide these definitions and proofs as most mathematical operations can be composed from basic operations that satisfy the axioms. We leave the definition and support of other arithmetic operators, such as exponentiation and roots, as future work.

All other operations and features supported by PUnits are built upon subtyping. For number comparison operations, PUnits requires that one of the two arguments be a subtype of the other. Similarly, it encodes a pair of subtype constraints for comparisons during inference. A comparison between a scientific unit and $\perp$ is permitted to support null reference comparisons, as $\perp$ is the type of null. Comparisons between different scientific units are forbidden as they are never subtypes of each other. For assignment operations, an equality constraint is generated between the expression and the refined target variable (see Sec. 4.1.2). Equality constraints are represented as a pair of subtype constraints where the two sides are subtypes of each other. Comparison and equality constraints are not introduced in Sec. 3.2 as they are built upon subtyping, but their encodings are described in detail in Sec. 4.3.

We believe that extending the formalization by explicitly modeling additional features of the Java language will not provide additional insights about the units relations other than, for example, the standard rules for behavioral subtyping.

\section{PUNITS DESIGN AND IMPLEMENTATION}

PUnits is implemented for Java. It is built on top of the Checker Framework [Dietl et al. 2011a; Papi et al. 2008] and Checker Framework Inference [Dietl et al. 2011b], and is openly available. ${ }^{4}$ The Checker Framework is a pluggable type system development framework for Java, allowing type systems to plug into the Java compiler to enforce stronger semantics. Checker Framework Inference is a framework which extends type systems developed on top of the Checker Framework with whole-program type inference capabilities, using solvers to solve typing constraint sets.

Sec. 4.1 explains PUnits's key features that reduce annotation effort. Sec. 4.2 presents the syntax for declaring base and derived units. Finally, Sec. 4.3 presents the constraint solving approach and the encoding of constraints.

\subsection{Type System Features}

PUnits supports annotation defaulting, method-local flow-sensitive type refinement, parametric polymorphism over units, and receiver-dependent units. These features minimize the need for unit annotations in method bodies, removing unnecessary clutter.

\footnotetext{
${ }^{4}$ https://github.com/opprop/units-inference
} 


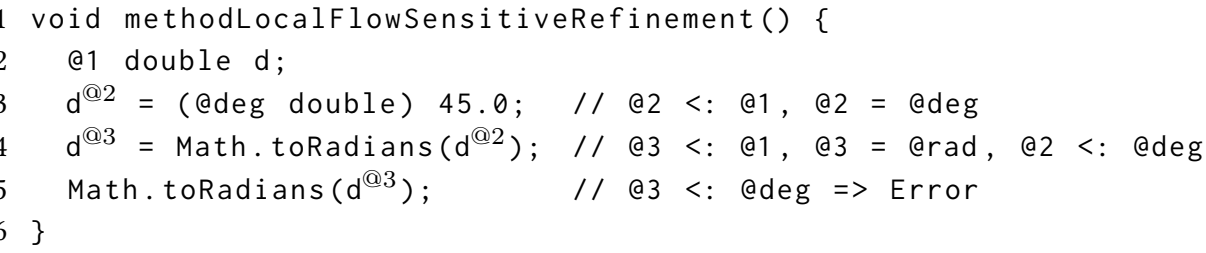

Fig. 8. Method-local flow-sensitive units refinement.

4.1.1 Defaulting Rules. The type rules forbid assignments and comparisons of values with incompatible units. To reduce the annotation burden in type check mode, the following default units are used for types with missing units:

- $T$ is the default unit for local variables.

- $\perp$ is the default unit for implicit and explicit lower bounds of type variables.

- QDimensionless is the default unit for all other type use locations, and the default unit of number literals.

Setting the default unit of local variables to T reduces the annotation burden, as the units of local variables are refined through flow-sensitive type refinement (Sec. 4.1.2).

Setting the default bounds of type variables to $T$ and $\perp$, respectively, allows users to instantiate generic data types and use generic methods with any unit, for example List<@N Float>.

Setting @Dimensionless as the default unit for all other type use locations restricts all flow of values with units in or out of unannotated formal parameters and returns of methods. It also forbids the use of methods with unannotated receiver parameter this on objects that are created with a unit, which prevents unintended use of methods that are incompatible with units.

4.1.2 Method-Local Flow-Sensitive Units Refinement. PUnits refines the unit of local variables through method-local flow-sensitive type refinement. If unannotated, the unit of a local variable starts as $T$ and is refined upon every assignment.

Method-local flow-sensitive refinement was not shown in Fig. 4 to avoid confusion. The example in Fig. 8 highlights the types and generated constraints. Constraint variables are introduced for types in variable declarations. @1 is the constraint variable for the declarations of $d$. During type checking, the types of local variables default to T. On line 3, a refinement type @2 is introduced for the type of $\mathrm{d}$ after the assignment. The unit of $d$ is refined from $T$ to @deg (degrees). The refined type of $d$ is safely used as an argument to toRadians() on line 4. Another refinement type @3 for $\mathrm{d}$ is generated as it is reassigned another value. The method toRadians() returns a arad value, which is stored in refinement type @3 of d. Note how the type of $d$ can change through these re-assignments. Line 5 causes an error, as toRadians () requires its argument to be @deg and the refined type of $d$ is now arad.

PUnits permits reusing local variables to store values with various units. However, it forbids the incorrect use of local variables. For each re-assignment of a local variable, a new refinement type is introduced. Each refinement type must be a subtype of the declared type of the variable and a corresponding subtype constraint is generated. Additionally, an equality constraint is generated between the expression type and the refinement type, to ensure the refinement type has the most specific type from the expression. 


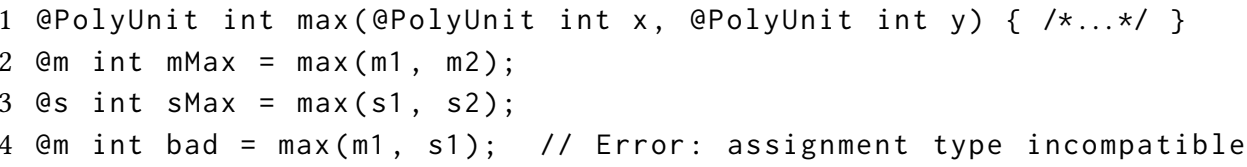

Fig. 9. Polymorphic method example. The variables $\mathrm{m} 1$ and $\mathrm{m} 2$ have the unit of @m (meters). The variables $\mathrm{s} 1$ and s2 have the unit of @s (seconds).

4.1.3 Parametric Polymorphism of Units. PUnits supports parametric polymorphism of units in two forms: using the special @PolyUnit type qualifier to parameterize methods, or using type polymorphism (Java generics) to parameterize methods or classes with units-qualified types.

PUnits supports parametric polymorphism of units for methods via the special type qualifier @PolyUnit. For example, a developer can declare a method that computes the maximum of any two values with corresponding units as shown in line 1 of Fig. 9. When this method is invoked with two variables in meters (line 2), it will return the maximum value with the unit of meters. When invoked with two variables in seconds (line 3), it will return the maximum value with the unit of seconds.

At every method call site, PUnits computes a unit which is used to instantiate @PolyUnit for the called method. The unit is computed as the least-upper-bound of the method receiver and/or argument units. The computed unit is $T$ for the call on line 4, because the arguments use different units @m and @s, and T is used as the return type of the method call. This reflects the fact that it is statically impossible to determine which of the two values gets returned and thus which unit is associated with the returned value. The assignment on line 4 gives an error, because it requires the result to be a meter.

PUnits utilizes polymorphic method signatures in inference and annotate modes, and generates the corresponding constraints. Fresh type variables are generated at each call site and constrained to be the least-upper-bound of the arguments. Within a method declaration, the parameters of polymorphic methods are checked by substituting $T$ for @PolyUnit, and the return is checked by substituting $\perp$ for @PolyUnit, since it can be instantiated with any unit.

PUnits does not infer additional @PolyUnit annotations during whole program inference, but uses existing polymorphic units correctly. None of the projects in our case study requires a method to be annotated with @PolyUnit as an inference result to satisfy constraints. We therefore did not need to design a strategy to infer @PolyUnit method signatures and kept the inference complexity small.

4.1.4 Receiver-Dependent Units. A return type or method parameter type sometimes needs to be sensitive to the actual method receiver type. PUnits supports receiver-dependent units by introducing the @RDU type qualifier for return types or method parameter types. A QRDU type is resolved using the actual receiver type, similar to how viewpoint adaptation works in ownership types [Dietl et al. 2007, 2011b]. The viewpoint adaptation operation takes two inputs, the receiver type and the declared type, and yields a single result type. When type checking a method invocation, any occurrence of @RDU in the signature is substituted with the receiver type; all non-@RDU-types stay unchanged.

Type checking happens against the viewpoint adapted signature of the method. @RDU is never inferred. In inference and annotate mode, any occurrence of QRDU in the signature introduces a new constraint variable and an equality constraint is introduced between the receiver type and the 


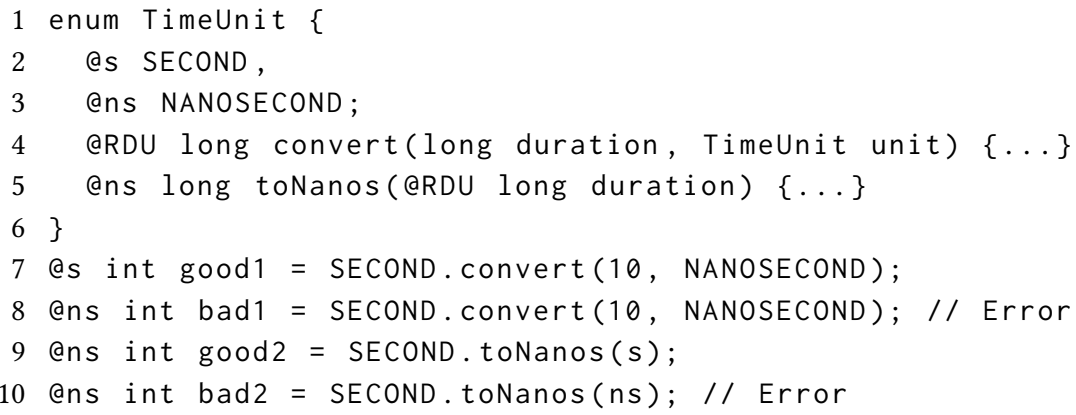

Fig. 10. Receiver-dependent units examples. Variable $s$ has unit type @s (seconds) and variable $n s$ has unit type @ns (nanoseconds).

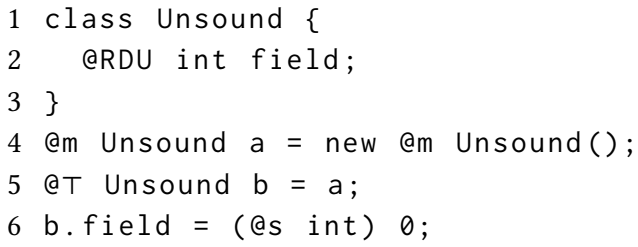

Fig. 11. Example of unsoundness if receiver-dependent units were allowed to be used on member fields. @RDU is forbidden on any program element aside from method parameter and method return types.

new constraint variable. This constraint variable is used for further checks instead of the declared parameter or return type.

Consider the example in Fig. 10. The return type of convert() on line 4 and the parameter type of toNanos() on line 5 are @RDU. When these methods are invoked, we enforce the return type of convert () and the argument to toNanos() to be the same as their method receivers. An invalid type assignment is issued on line 8 as SECOND. convert() returns type @s. An invalid argument type is issued on line 10 as SECOND. toNanos() accepts type @s as its argument.

Note that we forbid the use of $Q R D U$ on any program element aside from method parameters and method returns; otherwise soundness is not guaranteed. Fig. 11 shows an example of unsoundness if @RDU is used on a member field. At line 6, b. field is assigned a @s value, which is visible to a.field since object $b$ is an alias of object $a$, and breaks that a. field should be $@ m$.

\subsection{Declaring Units}

PUnits allows developers to declare a base unit by defining a Java 8 type annotation [Ernst et al. 2012] with the meta-annotation @BaseUnit. A meta-annotation is an annotation written on the declaration of another annotation. The annotation name of a base unit is used as its symbol.

Type annotation @Uni tsRep implements $T, \perp$, and the normalized representation. The annotation contains two boolean fields for $T$ and $\perp$, an integer field $\mathrm{p}$ for the base-10 prefix, and an array of @BUC annotations. The @BUC annotation contains an annotation class literal field to reference a base unit annotation, and an integer field to store an exponent. Each @UnitsRep instance is uniquely interpreted as either $\mathrm{T}, \perp$, or a scientific unit depending on the boolean and integer values. PUnits 
is appropriate for the inference of expressive type systems [Juma et al. 2020]. PUnits uses the MaxSMT solver because the constraints require integer theories for inferring exponents.

Each constraint variable $\alpha$ is encoded as two boolean variables $\beta^{\text {top }}$ and $\beta^{\text {bot }}$ which respectively represents $T$ and $\perp$, and one integer variable $\omega$ per exponent in the normalized representation, including the base-10 prefix exponent. For example, emPERs is encoded as $\neg \beta^{\text {top }} \wedge \neg \beta^{\text {bot }} \wedge \omega^{p}=$ $0 \wedge \omega^{m}=1 \wedge \omega^{s}=-1$, where $m$ and $s$ are the base units meter and second, and $p$ is the base-10 prefix. Each constraint presented in Sec. 3.2 is encoded as a predicate expressed over the boolean and integer variables.

The encoding of the hard constraints are:

- Well-formed Constraint $(w f(\alpha))$ : ensures each constraint variable represents either $\mathrm{T}, \perp$, dimensionless, or a concrete unit.

$$
\begin{aligned}
w f(\alpha):= & \left(\beta^{t o p} \wedge \neg \beta^{b o t} \wedge \omega^{z_{1}}=0 \wedge \cdots \wedge \omega^{z_{k}}=0\right) \vee \\
& \left(\neg \beta^{\text {top }} \wedge \beta^{b o t} \wedge \omega^{z_{1}}=0 \wedge \cdots \wedge \omega^{z_{k}}=0\right) \vee\left(\neg \beta^{t o p} \wedge \neg \beta^{b o t}\right)
\end{aligned}
$$

- Subtype Constraint $\left(T_{a}<: T_{b}\right)$ : given the type lattice, it is enough to check whether the subtype is the bottom type, the supertype is the top type, or all exponents are equal.

$T_{a}<: T_{b}:=\beta_{b}^{t o p} \vee \beta_{a}^{b o t} \vee\left(\omega_{a}^{z_{1}}=\omega_{b}^{z_{1}} \wedge \cdots \wedge \omega_{a}^{z_{k}}=\omega_{b}^{z_{k}}\right)$

- Comparison Constraint $\left(T_{a}<:>T_{b}\right)$ : two types are comparable if one is a subtype of the other. $T_{a}<:>T_{b}:=\beta_{a}^{t o p} \vee \beta_{b}^{t o p} \vee \beta_{a}^{b o t} \vee \beta_{b}^{b o t} \vee\left(\omega_{a}^{z_{1}}=\omega_{b}^{z_{1}} \wedge \cdots \wedge \omega_{a}^{z_{k}}=\omega_{b}^{z_{k}}\right)$

- Equality Constraint $\left(T_{a}=T_{b}\right)$ : two types are equal if they are subtypes of each other, which means that their encodings are equal.

$T_{a}=T_{b}:=\beta_{a}^{t o p}=\beta_{b}^{t o p} \wedge \beta_{a}^{b o t}=\beta_{b}^{b o t} \wedge \omega_{a}^{z_{1}}=\omega_{b}^{z_{1}} \wedge \cdots \wedge \omega_{a}^{z_{k}}=\omega_{b}^{z_{k}}$

- Addition Constraint $\left(\alpha_{c}=T_{a}+T_{b}\right)$ : the operation-subtyping consistency axiom (Fig. 7) guides our definition for the encoding of operations. The resulting type is an upper bound of the two arguments. Note that we do not require that it is a least-upper-bound.

$\alpha_{c}=T_{a}+T_{b}:=T_{a}<: \alpha_{c} \wedge T_{b}<: \alpha_{c}$

- Subtraction Constraint $\left(\alpha_{c}=T_{a}-T_{b}\right)$ : has the same encoding as addition.

- Multiplication Constraint $\left(\alpha_{c}=T_{a} * T_{b}\right)$ : if either argument is $\mathrm{T}$, the result has to be top. If one argument is $\perp$, while the other argument is not $T$, the result is $\perp$. If neither of the arguments is $T$ or $\perp$, the resulting exponents are added.

$$
\begin{gathered}
\alpha_{c}=T_{a} * T_{b}:=\left(\left(\beta_{a}^{\text {top }} \vee \beta_{b}^{\text {top }}\right) \wedge \beta_{c}^{\text {top }}\right) \vee\left(\beta_{a}^{\text {bot }} \wedge \neg \beta_{b}^{\text {top }} \wedge \beta_{c}^{\text {bot }}\right) \vee\left(\beta_{b}^{\text {bot }} \wedge \neg \beta_{a}^{\text {top }} \wedge \beta_{c}^{\text {bot }}\right) \vee \\
\left(\neg \beta_{a}^{\text {top }} \wedge \neg \beta_{b}^{\text {top }} \wedge \neg \beta_{c}^{\text {top }} \wedge \neg \beta_{a}^{\text {bot }} \wedge \neg \beta_{b}^{\text {bot }} \wedge \neg \beta_{c}^{\text {bot }} \wedge\right. \\
\left.\omega_{c}^{z_{1}}=\omega_{a}^{z_{1}}+\omega_{b}^{z_{1}} \wedge \cdots \wedge \omega_{c}^{z_{k}}=\omega_{a}^{z_{k}}+\omega_{b}^{z_{k}}\right)
\end{gathered}
$$

- Division Constraint $\left(\alpha_{c}=T_{a} \div T_{b}\right)$ : has the same encoding as multiplication except the resulting exponents are subtracted.

In annotate mode, PUnits generates additional breakable clauses that express preferences. The solver will attempt to satisfy all breakable clauses. The additional breakable clauses (enclosed in []) for the well-formed, subtype, and comparison constraints are:

- Well-formed Constraint $(w f(\alpha))$ : the breakable constraints prefer solutions that are units (that is, not $\mathrm{T}$ or $\perp$ ) and prefers dimensionless over other units. $w f(\alpha):=\ldots \wedge\left[\neg \beta^{t o p} \wedge \neg \beta^{b o t}\right] \wedge\left[\omega^{z_{1}}=0 \wedge \cdots \wedge \omega^{z_{k}}=0\right]$

- Subtype Constraint $\left(T_{a}<: T_{b}\right)$ : the breakable constraint prefers that the subtype and the supertype are equal.

$T_{a}<: T_{b}:=\ldots \wedge\left[T_{a}=T_{b}\right]$

- Comparison Constraint $\left(T_{a}<:>T_{b}\right)$ : the breakable constraint prefers that the two types are equal.

$T_{a}<:>T_{b}:=\ldots \wedge\left[T_{a}=T_{b}\right]$ 


\begin{tabular}{|l|l|c|c|c|c|c|c|c|}
\hline Project & Purpose & Files & SLOC & Arith & Comp & Time & Thread & Math \\
\hline Daikon & Invariant detection Tool & 734 & 168983 & 12671 & 16497 & 59 & 1 & 0 \\
\hline exp4j & Math Expression Evaluator & 30 & 5129 & 945 & 229 & 7 & 1 & 54 \\
\hline GasFlow & Gas Pipeline Graph Model & 189 & 15233 & 156 & 768 & 4 & 0 & 2 \\
\hline imgscalr & Image-scaling Library & 11 & 1178 & 18 & 79 & 12 & 0 & 3 \\
\hline jblas & Matrix Library & 74 & 11841 & 471 & 1055 & 7 & 1 & 39 \\
\hline JLargeArrays & 64-bit-index Arrays & 17 & 11337 & 868 & 2161 & 54 & 0 & 2 \\
\hline jReactPhysics3D & 3D Physics Engine & 105 & 10455 & 672 & 465 & 1 & 0 & 22 \\
\hline react & Real-time Physics Library & 63 & 10095 & 579 & 463 & 4 & 2 & 50 \\
\hline Total & 1223 & 234251 & 16380 & 21717 & 148 & 5 & 172 \\
\hline
\end{tabular}

Fig. 14. The purpose, size, and number of used arithmetic operators, comparison operators, and annotated JDK methods of the projects. The SLOC column counts the number of non-comment, non-blank lines of code. The Arith column counts uses of all arithmetic operators. The Comp column counts uses of all numeric comparison operators. The Time column counts uses of System. currentTimeMillis() and System. nanoTime(). The Thread column counts uses of Thread.sleep(). Finally, the Math column counts uses of all Math trigonometry methods.

We rely on the SMT solver to produce the optimal solution guided by hard and soft constraints provided to the solver. For example, the soft constraint added to the subtype constraint tells the solver to prefer the subtype (concrete units) instead of the supertype $(T)$. The annotation mode inserts the inferred solutions back into the source code, giving the developer a chance to inspect the results.

\section{EXPERIMENTS}

This section presents several experiments to evaluate PUnits's type checking and whole-program type inference capabilities. We answer three research questions:

RQ1: What are the benefits and trade-offs of using PUnits versus existing Java unit libraries?

RQ2: Can PUnits detect and prevent units-related errors in real-world projects?

RQ3: What is the compilation overhead of PUnits in each of its three modes?

To answer these research questions, we provide annotated JDK specifications, including the Math trigonometry methods, System time methods, and Thread sleep methods. In total, we add 33 unit annotations to 18 JDK methods. We also provide 39 methods in the Java Math library and 81 boxed primitive methods with polymorphic units and 8 methods in the TimeUnit library with receiver-dependent unit types. These methods are selected because they are used in a wide range of projects and are prone to misuse.

The benchmark consists of open-source Java scientific-computing projects that utilize the annotated methods, and we can successfully build them (unfortunately this is not true for all open-source projects, due to bit-rot, lack of documentation, etc.). In total, we selected eight projects. Some projects are small but they perform interesting arithmetic operations. Fig. 14 summarizes the size and purpose of each project, together with the number of used arithmetic operators, numeric comparison operators, and annotated JDK methods. The size of the projects ranges from 538 to 168983 lines of source code, totalling 234251 lines. 


\subsection{RQ1: What are the benefits and trade-offs of using PUnits versus existing Java unit libraries?}

We discuss the benefits and trade-offs from three aspects: error detection, program execution performance (time and memory), and features.

In this RQ, we focus on analyzing PUnits's effects on the GasFlow project, the only project that uses a unit library. GasFlow uses the JScience library, or JSR 275/363 [Dautelle and Keil 2010; Dautelle et al. 2016], which is one of the most popular unit libraries for Java. We replace uses of JScience unit wrapper classes with PUnits specifications and primitive types and run modular type checking on the annotated code.

The JScience API uses generics to provide unit type-safety. JScience uses the Amount<Quantity ${ }^{5}>$ class for storing the exact Unit ${ }^{6}$ and performs arithmetic operations with the units. To replace the abstract data types with primitives, we create helper class UnitsTools, which consists of units that are represented using annotated primitives with a value of 1 (eg. @m int $\mathrm{m}=1)$. UnitsTools also contains conversion methods that are used to replace unit conversion functions in JScience when it is possible to determine the type to be converted statically. All the unit conversions that appear in the GasFlow project can be determined statically. A detailed description of the JScience library and a breakdown of the units and functionalities replaced are described in Appx. C. Fig. 15 shows an example of a converted function. In total, 510 JScience method invocations and 503 variables and method declarations containing 17 dimensions and 22 units are replaced with 647 PUnits qualifiers and 242 UnitTools uses. Additional PUnits qualifiers were needed for casting unit-wise heterogeneous methods to specific units.

5.1.1 Error Detection. After replacing units wrappers with PUnits specifications and primitive types, we used PUnits in type checking mode to see whether there are any units errors. We found three units errors, whereas JScience failed to detect them. The reason why JScience cannot detect these errors is due to the use of casting and raw types. The original GasFlow contains 130 raw unit data types and 51 unit casts. Line 3 in Fig. 15 illustrates an illegal cast of an area to a length. The return type of times() is Amount<?>, because JScience cannot statically express the return unit. Developers are therefore required to add casts that cannot be checked statically, to make the result usable. Similarly, on line 4, a raw type is used to circumvent this weakness. The cast from a wildcard to a type produces an unchecked warning, as does the usage of a raw type. 181 such warnings are produced in GasFlow. Making matters worse, no runtime exception is raised within method bad(), as all method invocations and casts are valid. However, even though the type argument is incorrect, the unit is still preserved dynamically. If we try to read the return value of $\operatorname{bad}()$ in a unit that is in the dimension of the signature on line 1, such as meter or kilometer, a runtime exception will be raised. As such invocations can happen a long time after the call to $\operatorname{bad}()$, it is difficult to pinpoint where the incorrect Amount object came from.

With PUnits, this kind of casting will not be allowed since $\mathrm{m}$ is not a subtype of $\mathrm{m}^{2}$. Not only that, with PUnits, such casts are not needed. Variable $a$ has type $\mathrm{m}^{2}$ and it is not a subtype of $\mathrm{m}$. An invalid cast error will be issued on line 9 and an invalid return type error will be issued on line 11 . PUnits is able to detect errors early and it pinpoints the exact location of the problem.

5.1.2 Execution Time \& Memory Consumption. We executed the GasFlow project by invoking the main method of the program. The program calculates and generates data points at every time step over a fixed amount of time. The time step was originally set to 1 second. To better analyze its performance, we decrease the time step to 1 millisecond to increase the program execution time.

\footnotetext{
${ }^{5}$ Part of the javax.measure package, provides dimension handling.

${ }^{6}$ Part of the javax. measure package, provides unit handling.
} 


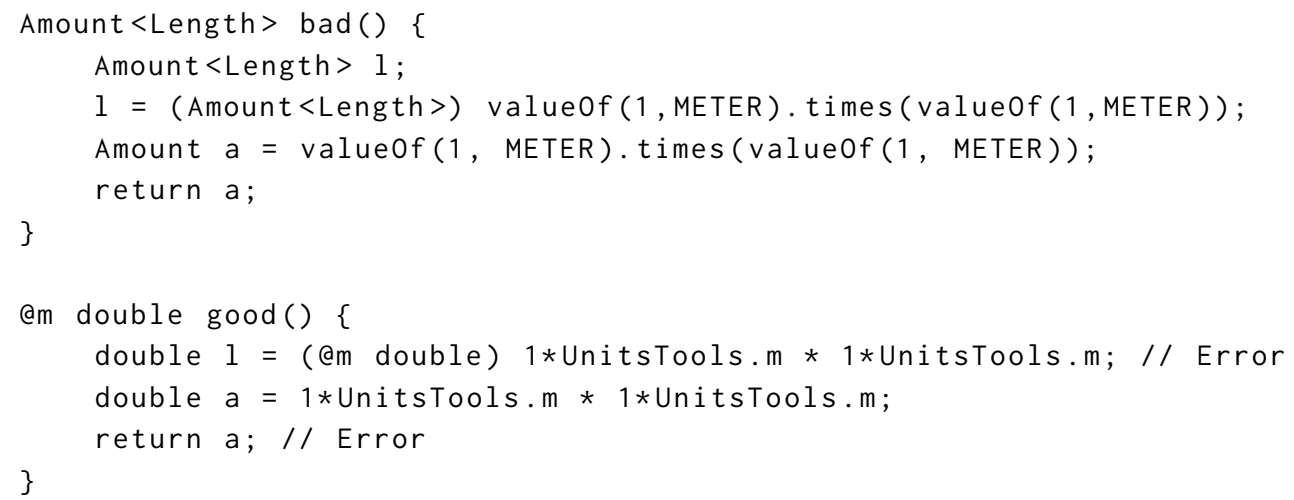

Fig. 15. A simple example comparing the difference between JScience and PUnits.

This case study is performed on a cloud instance with a four-core CPU and 16GB RAM, running 64-bit Ubuntu 20.04.

The average execution time is $3966 \mathrm{~ms}$ when we are using JScience, and $3367 \mathrm{~ms}$ when using PUnits and all the abstract data types are replaced with primitives. The execution time has significantly reduced by $15.1 \%$. The execution time is measured using Java's System library.

The average memory consumption is 794 kBytes when we are using JScience, and 697 kBytes when using PUnits with primitive types. The memory consumption has significantly reduced by $12.2 \%$. The memory consumption is measured using Java's Runtime library.

The performance overhead of UnitsTools is insignificant compared to JScience, as all of the UnitsTools operations are using primitives. As PUnits is a static analysis tool, it will not add any overhead to the program during runtime and reap the benefits of using primitives, unlike JScience which uses abstract data types to keep track of units.

5.1.3 Features. GasFlow contains one heterogeneous method, which accepts or returns a range of dimensions that differ in its specific class or type argument. For this heterogeneous method, PUnits loses the unit of the method parameter and return variable, while JScience preserves the unit dynamically through abstract data types. Nevertheless, we believe it is good coding practice to avoid type-unsafe heterogeneous methods when possible, as heterogeneous methods increase the risk of a runtime exception. PUnits enforces this programming practice.

PUnits uses primitives instead of abstract data types, which also prevents problems with null values. As a value should always have a unit, we do not see this as a restriction. One simple way to allow the primitives to have a null-like behaviour is by using boxed primitives, or creating a boolean flag for those variables to indicate if it has been initialized or not. In GasFlow, there are five variables that required this work-around.

It is possible to combine PUnits with a units library, by annotating the abstract data types of the units library with PUnits specifications to gain the benefits of both static error detection and dynamic library features. However, this will lose the performance benefits of using primitive types. Overall, for projects that require minimum uses of dynamic unit computations, PUnits is a better alternative than JSR 275/363. 


\subsection{RQ2: Can PUnits prevent units-related errors in real-world projects?}

PUnits is able to type check all eight projects. In total, 97 errors were issued as the projects were unannotated and flows were detected which propagate units from annotated methods to defaulted aDimensionless method parameters or returns. These errors are expected from unannotated projects.

The Firefox Android code at the commit where the error from Fig. 1 was introduced no longer builds, due to many of the project's dependencies no longer being available. We run PUnits on a minimized snippet extracted from the projects, which reveals the reported error presented in Fig. 1.

GasFlow is fully annotated, originally with JScience units types, which we then translated to PUnits specifications. Therefore, we only ran modular type checking on it, revealing three errors. Two of these errors are related. Function computeSiamCoefficient is declared as dimensionless. However, the return type is $m^{-1} s^{-2}$ and an invalid return error is issued. This function is then invoked by a function that requires the return type to be $\mathrm{m}^{-1} \mathrm{~s}^{-2}$, but as computeSiamCoefficient is declared as dimensionless, another return type error is issued. We changed the return type to $m^{-1} s^{-2}$ to fix these two errors. The other error is related to function getReynoldsNumber. A Reynolds Number [Benson 2014] is a dimensionless value, but the function returns type $\mathrm{m}^{3} / \mathrm{kg}$. This function is missing the density (volume per weight) variable to give the correct Reynolds Number. This error causes all future computations in this project to be incorrect.

Daikon at the commit containing both unit errors, [Daikon 2003, 2004], cannot be compiled successfully using modern versions of the tools, as the newer versions generated syntactically invalid source code. We decided to insert the errors back into the current source code to see whether or not PUnits can detect them. The file containing the first error [Daikon 2003] no longer exists, so only the second error [Daikon 2004] is inserted. We manually annotated the project and type checked the program. PUnits successfully detected the second error [Daikon 2004] and issued an argument incompatible error. We fully annotated the project with 27 scientific units and can now guarantee that these kinds of errors will not be introduced again.

For the other six projects with no known errors, we use PUnits to infer and fully annotate the projects. PUnits is able to infer and annotate units for three of the projects without modifications. The other three projects reached unsatisfiable constraints in inference, and we analyzed them further to determine the root issues. The findings are discussed in Sec. 5.2.1 below. For two of the unsatisfiable projects, we addressed the diagnosed issues and subsequently inferred and annotated units. Fig. 16 summarizes the number of variables and constraints generated for the projects, the number of SMT variables and formulas encoded for the constraint system, and the units inferred in annotate mode. The annotate mode results for the two successfully patched projects are shown in Fig. 17.

We manually inspected the annotate mode results. The majority of the scientific units are inferred for the types of variables and method parameters. The single $T$ unit is inferred for a logging method's parameter. The method is inspected and the annotation is deemed correct as the method is unit-wise agnostic. All of the @Dimensionless annotations are inferred for variables and parameters that do not interact directly or indirectly with the annotated JDK methods. PUnits does not insert a unit into source code if it matches the default unit for the corresponding type use location. The numerous @Dimensionless units in the results are not inserted into the source code.

The overall annotation effort is very low. Given 33 manually added JDK unit annotations, PUnits was able to infer and annotate 25 scientific units for the three successful projects and 62 units for the two patched projects. The number of inferred units depends on the number of annotations manually added, on how often the annotated variables and methods are used, and on how interconnected the constraints expressed over the variables are. However, note that simply

Proc. ACM Program. Lang., Vol. 4, No. OOPSLA, Article 142. Publication date: November 2020. 


\begin{tabular}{|l|c|c|c|c|c|c|c|c|c|c|c|c|c|c|c|}
\cline { 2 - 15 } \multicolumn{1}{c|}{} & \multicolumn{3}{c|}{ PUnits } & \multicolumn{4}{c|}{ SMT } & \multicolumn{5}{c|}{ Inferred Units } \\
\hline Project & $\alpha$ & $\mathrm{E}$ & $\Sigma$ & Bool & Int & Assert & $\begin{array}{c}\text { Soft } \\
\text { As- } \\
\text { sert }\end{array}$ & $T$ & D & ms & ns & deg & rad & rad $^{-1}$ \\
exp4j & 1438 & 1 & 1495 & 5752 & 2876 & 8501 & 2733 & \multicolumn{5}{c|}{ UNSAT } \\
\hline imgscalr & 841 & 3 & 994 & 1682 & 3364 & 3561 & 1750 & 1 & 542 & 12 & 0 & 3 & 0 & 0 \\
\hline jblas & 17506 & 2 & 22147 & 70024 & 105036 & 117930 & 38728 & \multicolumn{5}{c|}{ UNSAT } \\
\hline JLargeArrays & 12586 & 2 & 14017 & 50344 & 75516 & 78087 & 25181 & \multicolumn{6}{c|}{ UNSAT } \\
\hline jReactPhysics3D & 9499 & 1 & 14899 & 18998 & 18998 & 46660 & 22398 & 0 & 5798 & 0 & 1 & 0 & 0 & 0 \\
\hline react & 12693 & 2 & 17729 & 25386 & 38079 & 58337 & 28024 & 0 & 8544 & 0 & 1 & 0 & 5 & 3 \\
\hline Total & 54565 & 73452 & 109130 & 243869 & 313076 & 118811 & 1 & 14884 & 12 & 2 & 3 & 5 & 3 \\
\hline
\end{tabular}

Fig. 16. Annotate mode results for the projects. Columns $\alpha$ and $\Sigma$ show the number of constraint variables and constraints generated by PUnits. Column E shows the number of base units encoded for each constraint variable. Column Assert shows the number of (mandatory) formulas encoded for SMT and MaxSMT. Column Soft Assert shows the number of breakable formulas encoded for MaxSMT. The units shown are the solutions given by PUnits in annotate mode. Note that $\perp$ did not appear in the solutions for any of the projects. D stands for @Dimensionless.

\begin{tabular}{|c|c|c|c|c|c|c|c|c|c|c|c|c|c|c|}
\hline & \multicolumn{3}{|c|}{ PUnits } & \multicolumn{4}{|c|}{ SMT } & \multicolumn{7}{|c|}{ Inferred Units } \\
\hline Project & $\alpha$ & $\mathrm{E}$ & $\Sigma$ & Bool & Int & Assert & Soft Assert & $T$ & $\mathrm{D}$ & $\mathrm{ms}$ & ns & deg & $\mathrm{rad}$ & $\mathrm{rad}^{-1}$ \\
\hline exp4j & 1480 & 1 & 1465 & 2960 & 1480 & 5683 & 2776 & 0 & 955 & 0 & 0 & 0 & 20 & 0 \\
\hline jblas & 17537 & 2 & 22171 & 35074 & 52611 & 78431 & 38770 & 0 & 12687 & 4 & 6 & 0 & 35 & 0 \\
\hline Total & 19017 & & 23636 & 60038 & 84114 & 41546 & 65624 & 0 & 13642 & 4 & 6 & 0 & 55 & 0 \\
\hline
\end{tabular}

Fig. 17. Annotate mode results for the patched projects. The number of constraint variables, constraints, SMT variables and formulas vary slightly compared to the numbers reported in Fig. 16. This is due to the code changes introduced in the patches.

adding more JDK unit annotations would not make the inference problem harder: the same set of constraints would be generated for the programs and different solutions would be found. Using the 33 JDK annotations gives a good impression of the inference approach, even if most inferred annotations are dimensionless.

5.2.1 Unsatisfiable Inference Scenarios. Projects exp4j, jblas, and JLargeArrays failed to initially infer any solutions as they contain unsatisfiable constraints. We investigated the code locations which generated the unsatisfiable constraints. The following discusses the insights we learned from the analysis and from the efforts to patch the code.

$\exp 4 \mathbf{j}$ is a mathematical expression evaluator for Java. The program allows users to declare variables and specify a complex expression, including calls of math library functions, in a string, for example, $" \sin (x)-\log (3 * x / 4) "$. The program evaluates these strings and gives the user a final answer. Math library functions are abstracted through method double apply(double... args). The functions are recursively applied to partial answers according to the user input until a final answer is computed. Each implementation calls a concrete math library method, some of which we had annotated with unit annotations.

The project reached UNSAT in inference due to mismatches in the expected parameter and return units of apply(). Some implementations expect a @rad argument and others expect a 
@Dimensionless argument. As math functions return results in different units, the common abstraction requires the return type of $\operatorname{apply}()$ be $T$. Due to the recursive application of the functions, the parameter also has to be typed $T$. This then results in an unsatisfiable constraint set given the JDK math annotations.

Fundamentally, the dynamic evaluation of the input string prevents static checking of the unit annotations. Encapsulating values in this program using JSR 363's units API [Dautelle et al. 2016] would check the units of values at run-time.

We patched this project by always treating computed answers as @Dimensionless and suppressing mismatches when evaluating the expression string. The inferred units annotate the trigonometry functions with a parameter or return type of radians.

jblas is a matrix library for Java. It provides a number of floating point matrix abstractions and defines mathematical functions for matrices. In particular, classes DoubleMatrix and FloatMatrix each define the overloaded methods get (i) and put (i, v) which returns or inserts a value at index $i$ in the matrix, traversing row-wise first. Internally, the classes store matrix values in a one-dimensional array. Class MatrixFunctions defines methods to perform in-place updates to the matrix.

The project reached UNSAT because the operations read and write to the same array even if the values have different units. Although the numeric data type of the array is fixed, the array is used in a unit-wise heterogeneous way in which there is no possible typing with a unit. Users of this library will need to be careful with the order of using the methods. To solve this fundamental issue, the library would need to be rewritten to construct and return new matrices with different units instead of performing in-place updates.

We introduced a simpler, non-ideal, patch by always storing @Dimensionless values in the array, and casting the units as needed before they are passed to a math method. The inferred units annotate the return types and parameter types of the trigonometry methods in MatrixFunctions with radians, and the variables in a timer class with time units.

JLargeArrays defines arrays that can store up to $2^{63}$ elements. This project reached UNSAT through two different scenarios.

For the first scenario, the project uses low-level unsafe methods provided by the JDK to store and retrieve array values in order to support 64-bit indexing. Operations within the project can store @rad values into memory. If unannotated, JDK and external library methods are treated as @Dimensionless in inference mode. The flow of arad into the unannotated library methods causes an unsatisfiable typing. Six additional $T$ annotations are added to the low-level JDK method parameters to focus the inference on detecting problems in the project's code.

For the second scenario, the project defines superclass LargeArray which declares common methods such as Object get (long i) and void set(long $i$, Object $v$ ) to provide access to array elements. A method converts arrays from one subclass to another by repeatedly executing the code out.set(i, src.get(i)) to copy values. The project reached unsat in inference as some subclasses return @rad values for get (), and some subclasses expect @Dimensionless values passed to set(). The conversion method should also be refactored to be precise in terms of the types of arrays it reads from and writes to, so that a flow of values with incompatible units does not occur.

We did not fix this project as handling the get() and set() methods correctly requires a significant refactoring.

Overall, two reasons cause a project to reach UNSAT in inference mode:

(1) Presence of units errors. Projects exp4j, jblas, and JLargeArrays do not make any attempts to prevent a user from mistakenly passing values of one unit into a function that expects a 


\begin{tabular}{|c|c|c|c|c|c|c|c|c|}
\hline \multirow[b]{2}{*}{ Project } & \multirow[b]{2}{*}{$\begin{array}{l}\text { OpenJDK } \\
\text { Compile }\end{array}$} & \multirow[b]{2}{*}{$\begin{array}{l}\text { Type } \\
\text { Check }\end{array}$} & \multicolumn{4}{|c|}{ Inference } & \multicolumn{2}{|c|}{ Annotate } \\
\hline & & & Encode & Solve & $\begin{array}{l}\text { UNSAT } \\
\text { Encode }\end{array}$ & $\begin{array}{l}\text { UNSAT } \\
\text { Solve }\end{array}$ & Encode & Solve \\
\hline exp4j & 4.19 & 15.74 & 2.74 & 7.57 & 3.03 & 0.66 & 3.09 & 7.11 \\
\hline imgscalr & 3.65 & 12.30 & 2.85 & 2.68 & \multicolumn{2}{|c|}{$\mathrm{N} / \mathrm{A}$} & 3.62 & 33.89 \\
\hline jblas & 6.73 & 57.23 & 23.32 & 599.63 & 34.84 & 36.25 & 29.77 & 7576.25 \\
\hline JLargeArrays & 5.11 & 38.00 & 16.86 & 32.19 & 16.06 & 25.28 & \multicolumn{2}{|c|}{ No Patch } \\
\hline jReactPhysics3D & 4.68 & 41.59 & 22.91 & 184.15 & \multicolumn{2}{|c|}{$\mathrm{N} / \mathrm{A}$} & 29.84 & 771.84 \\
\hline react & 5.52 & 47.30 & 24.38 & 456.92 & \multicolumn{2}{|c|}{$\mathrm{N} / \mathrm{A}$} & 30.25 & 2149.77 \\
\hline Total & 29.88 & 212.16 & 93.06 & 1283.14 & 53.93 & 62.19 & 96.57 & 10538.86 \\
\hline
\end{tabular}

Fig. 18. Performance of PUnits running in the three modes. All values are in seconds. OpenJDK 11 compilation times for the projects are presented for comparison to type check mode performance. The type check times reported are for checking the entire project. The Encode and Solve columns, respectively, report the time taken by PUnits to encode a constraint system into SMT formulas, and for Z3 to solve the formulas. The Encode column does not count the time required to traverse the AST to generate constraints.

different unit. These errors can lead to serious failures if the libraries were used incorrectly in mission-critical systems. PUnits helps identify such fundamental design issues and forces developers to restructure their applications in a way that allows the safe use of units.

(2) Insufficient annotations for library methods. Applications have large dependencies on binaryonly libraries, like the JDK. PUnits can infer annotations for the available source code, but requires manual annotations for binary-only dependencies. PUnits can use optimistic defaults, for example by assuming that unannotated method signatures have $T$ receiver and parameter types, and $\perp$ return types. Optimistic defaults can temporarily help a developer pinpoint whether the problem is in their code or is due to an unannotated API. Manual annotation efforts for libraries can be re-used to type check and infer units in other projects utilizing the same APIs.

PUnits inferred 90 scientific units and generated well-specified applications for projects exp4j, imgscalr, jblas, jReactPhysics3D, and react. The annotations are available for human inspection ${ }^{7}$ to ensure specification correctness. In summary, PUnits is able to both detect and prevent unit related errors in real-world projects.

\subsection{RQ3: What is the compilation overhead of PUnits in each of its three modes?}

This case study is performed on a cloud instance with a four-core CPU and 16GB RAM, running 64-bit Ubuntu 20.04.

Fig. 18 reports the arithmetic mean of the execution times of PUnits in each of its three modes across 5 executions for the six projects. These projects take on average $4.98 \mathrm{sec}$ to compile (29.88 sec total) using the OpenJDK 11 compiler, and on average $35.36 \mathrm{sec}$ to type check (212.16 sec total) using PUnits in type check mode. The approximately $7.1 \mathrm{x}$ overhead is consistent with other type systems developed using the Checker Framework [Chen and Dietl 2018; Dietl et al. 2011a].

In inference mode, the projects took $1376.2 \mathrm{sec}(23 \mathrm{mins})$ to check and infer a type-safe solution. The overhead is approximately $6.49 \mathrm{x}$ compared to type check mode. For small projects, PUnits takes more time to generate SMT encodings than it takes for the solver to solve the constraints. For large projects such as react, the major performance bottleneck is the SMT solver.

\footnotetext{
${ }^{7}$ https://github.com/opprop/units-inference/tree/master/benchmarks
} 
In annotate mode, successful projects take $10635.84 \mathrm{sec}(177 \mathrm{mins})$ to infer precise units. jblas and react contribute the most to the tally. The overhead is approximately $50.13 \mathrm{x}$ vs. type check mode, and 8.01x vs. inference mode. The time is spent by the SMT solver to optimize the solutions.

The performance of PUnits in type check mode enables it to be used in edit-compile-unittest development workflows. As type check mode is modular, faster performance is expected when checking one source file at a time. The performance in inference mode for whole-program satisfiability checking enables it to be used in continuous integration workflows. The performance in annotate mode is slow, but this mode is expected to be used less frequently. The annotations inserted into source code enable subsequent uses of PUnits in inference or annotate modes to be executed faster, as fewer constraint variables are generated. Developers can also incrementally infer and annotate their projects, starting with core libraries.

Overall, PUnits performs adequately in each of its three modes. Its performance is suitable for use in a real-world software development environment. Improvements to the Checker Framework and Checker Framework Inference will improve the performance of PUnits and other type systems developed using the frameworks.

\section{RELATED WORK}

Two general approaches have been applied in prior work to add support for units to a programming language: (1) through designing abstract data types and libraries to encapsulate numbers with units, (2) through modifying a language to add units syntax and static analysis.

\subsection{Encapsulation Approach}

The encapsulation approach uses the existing type system of a language to perform limited static analysis, with the capability to perform fully run-time-based unit analysis for units and quantities that are given as run-time inputs. The design and features of 38 different units libraries are extensively compared in a recent paper [Bennich-Björkman and McKeever 2018]. Using such libraries incurs additional performance and memory overhead. For example, using boxed number types for numeric computations in Java is $3 x$ slower and uses $3 x$ more memory compared to using primitive types [Melzer 2015]. Units of measurement libraries exist for all programming languages, from Ada [Gehani 1977, 1985] and C++ [Schabel and Watanabe 2010] to Fortress [Allen et al. 2004] and .NET [Larsen 2018], and many, many others.

JSR 275/363 [Dautelle and Keil 2010; Dautelle et al. 2016] is the most popular option for Java. It defines an API for units of measurement and provides a reference implementation for SI units. The benefits and trade-offs of using PUnits versus such libraries are discussed in Sec. 5.1.

\subsection{Static Approaches}

The static approaches in prior work differ in how units are internally represented and their expressiveness, how types are inferred, and how annotation burden is reduced. Fig. 19 gives an overview of the supported features between the related works.

Osprey is a constraint-based units type checker for C [Jiang and Su 2006]. PUnits's representation of units is similar to Osprey's. Osprey introduces a units language whereby compound units are represented as products and inverses of SI base units, a "dimensionless" unit, and constant factors. Osprey does not allow developers to utilize a different set of base units, and stores only the exponents of the SI units. It also does not have a $T$ or $\perp$ type. There is no subtyping between units in Osprey. Assignments, additions, subtractions, and comparisons all require the arguments' units to be equal to each other while PUnits allows more flexibility with $T$ and $\perp$. Any return value and parameter without annotations are treated by Osprey to be polymorphic while in PUnits, functions that are intended to be polymorphic must be explicitly annotated. Osprey's minimized constraint 


\begin{tabular}{|l|c|c|c|c|c|c|c|}
\cline { 2 - 8 } \multicolumn{1}{c|}{} & PUnits & Osprey & F\# & Simulink's & B's & ableC's & CPF[UNITS] \\
\hline Dimension analysis & $\checkmark$ & & $\checkmark$ & $\checkmark$ & & & $\checkmark$ \\
\hline Unit-wise analysis & $\checkmark$ & $\checkmark$ & $\checkmark$ & & $\checkmark$ & $\checkmark$ & $\checkmark$ \\
\hline Support non-SI & $\checkmark$ & & $\checkmark$ & & $\checkmark$ & & $\checkmark$ \\
\hline Polymorphism & $\checkmark$ & $\checkmark$ & $\checkmark$ & $\checkmark$ & & $\checkmark$ & \\
\hline Receiver dependent & $\checkmark$ & & & & & & \\
\hline T/ $\perp$ (top/bottom) & $\checkmark$ & & & & $\checkmark$ & & \\
\hline$<$ kN/km> =<N/m> & $\checkmark$ & $\checkmark$ & & $\checkmark$ & & & $\checkmark$ \\
\hline Modular type inference & $\checkmark$ & $\checkmark$ & $\checkmark$ & $\checkmark$ & $\checkmark$ & & $\checkmark$ \\
\hline Whole-program inference & $\checkmark$ & & & & & & \\
\hline Reduce annotation burden & $\checkmark$ & $\checkmark$ & $\checkmark$ & & & & \\
\hline
\end{tabular}

Fig. 19. An overview of features provided by each unit of measurement system.

set is translated into a system of linear equations and then solved using Gaussian elimination. We also implemented a Gauss-Jordan elimination solver for the integer subsets of the constraints. However, the initial performance results of this solver did not look promising and we did not pursue the effort further. To reduce annotation burden, Osprey can suggest "critical" variables for users to annotate. These are variables whose units are inconsistent with the units of their representatives. In PUnits, if inference fails, the set of constraints causing unsatisfiability is provided. The user can examine the constraints and determine whether there is an unit error or there are insufficient annotations in the binary-only libraries.

F\#'s units type system [Kennedy 2009, 1997; Wlaschin 2012] represents units as symbols or products of symbols, each with an optional integer exponent. The set of symbols can be declared by the developer, however there is no support for representing prefixed units in terms of their base units, as prefixed units are considered distinct unit symbols. Consequently, a value with unit $<\mathrm{kN} / \mathrm{km}>$ cannot be assigned to a variable with unit $\langle\mathrm{N} / \mathrm{m}>$ in F\# whereas it is permitted in PUnits. F\# infers under-constrained type variables as polymorphic unit types, the most general typing in $\mathrm{F} \#$. In PUnits, the most general type is $\mathrm{T}$, which is not a useful annotation to insert into source code. PUnits chooses to infer the most precise type, and prefers to infer @Dimensionless for under-constrained type variables. Similar to PUnits, functions that are intended to be polymorphic must be explicitly annotated. F\# allows polymorphic functions to express more rich relationships such as polymorphic multiplication and division where the result unit is a function of two or more polymorphic units given as the parameters. PUnits currently supports a less expressive form of polymorphism, but scaling the current design to support F\# style polymorphism is interesting future work. We did not need F\# style polymorphism in the case studies, but plan to extend our implementation as future work.

F\#'s inference algorithm was improved upon in a type system for Fortran [Hangal and Lam 2009; Orchard et al. 2015]. This system makes two improvements. First, it always assumes that functions without any unit annotations are implicitly polymorphic over the units. It infers the unit relationships between a function's parameters and its return as a general relationship, and then infers the specific units at each call-site. Second, under-constrained variables are reported as critical variables which require manual annotation by a user. Critical variables provide the maximal amount of unit information with the minimal number of explicit annotations. This improvement reduces some annotation burden. PUnits inserts all inferred non-default units into source code, reducing the annotation burden for subsequent type checking or inference. 
A dimensional analysis system for Simulink [Owre et al. 2012] expresses dimensions as products of SI base dimensions, each with an integer exponent. Dimensional consistency can be enforced through this representation. However, unit-wise consistency cannot be enforced: an expression of 1 $\mathrm{km}+1 \mathrm{~m}$ would not result in any errors as both are in the dimension of length. A proper calculation would require either unit to be converted prior to the addition. This system infers dimensions by collecting equations from the program and then solving the set of equations through Gauss-Jordan elimination.

A units type system for the B language [Krings and Leuschel 2013] represents units as products of SI units where each base unit has a prefix and an exponent. Some derived units can be expressed via multiple representations in this design. For example, a kilo-newton can be defined as both $\mathrm{Mg} *$ $\mathrm{m} * \mathrm{~s}^{-2}$ and $\mathrm{kg} * \mathrm{~km} * \mathrm{~s}^{-2}$ with corresponding triples. This leads to a combinatorial explosion of representations since multiplication and division produce new units as a function of the units of its two arguments. PUnits extracts all prefixes into one constant, forcing each unit to have a unique normalized representation, which avoids the combinatorial explosion problem. This type system does not handle polymorphism and all unannotated units are treated as $\perp$. It uses a simple unification-based type inference, in addition with constraint solving for multiplication, division, and exponentiation operations. These constraints cannot be collected and are solved one at a time. The $T$ qualifier in this unit type system is used to indicate a type error as only one inferred unit is acceptable. In PUnits, $T$ is accepted to accommodate heterogeneous methods and arrays.

Type Qualifiers as Composable Language Extensions [Carlson and Van Wyk 2017] proposes adding pluggable type checkers to the ableC [Kaminski et al. 2017] language as grammar and type checking extensions, including a units of measurement system. In that system, units are parsed as symbols as part of an extended ableC grammar, and then represented as a set of tuples similar to the units type system for the B language[Krings and Leuschel 2013] for its type checking phase. Each tuple is of the form $\left[b^{p} * u^{e}\right]$ for some conversion factor $b$ raised to the power of $p$ and some base unit $u$ raised to the power of $e$. In $a b l e C$, units are not organized in a type lattice and there is no concept of $T$ or $\perp$ types. ableC's units type system is implemented exclusively for SI units, and does not support other units of measurements without further extending the grammar and implementing the extension. The pluggable type systems of ableC lack type inference capabilities.

CPF[UNITS] [Hills et al. 2012] defines a units analysis policy, annotation language, and specification that plugs into the $\mathrm{C}$ Policy Framework (CPF) to debug and verify $\mathrm{C}$ programs. The system encodes the abelian group properties of units as a rewriting system. Units are represented as products of base units, each with an exponent. The set of base units is customizable and can be given long and short names. Users provide annotations which specify the units of variables, objects, and function parameters and returns as pre- and post-conditions. Unannotated function parameters and objects are treated as having a "fresh" unique unit by the system to eagerly prevent misuses. The framework extracts one verification task per function. The tasks are solved through symbolic execution, using the rewriting system to solve units relationships. Units are checked at key operations such as additions and comparisons. The system is modular, and can infer units for local variables. However, it does not perform whole-program inference.

\section{CONCLUSION AND FUTURE WORK}

This paper presents $P U n i t s$, a pluggable type checking and whole-program type inference system for units of measurement. Three usage modes provide fast modular type checking, whole-program satisfiability checking, and whole-program inference of precise annotations that can be used for further analyses. PUnits is formalized and proven sound. PUnits is evaluated in Java, showing PUnits can reap the benefits of using primitives over abstract data types, and prevent units-related errors in real-world projects. PUnits requires only a few manual annotations to infer units, and manual 
effort can be reused when annotating additional projects. PUnits strikes a novel balance between expressiveness, inference complexity, and annotation effort. It is a practical tool for improving the quality and efficiency of software.

The work in this domain is not yet finished. The type inference approaches employed thus far either assume that unannotated method parameters and returns are polymorphic over units, or require explicit annotations to indicate polymorphism. No approach to date can infer that a method should be polymorphic over units. PUnits is the first units type system to adapt the concept of receiver-dependent types. PUnits currently does not infer whether a method parameter or return type should be receiver dependent over units. Finding an efficient encoding is left as future work.

Implementing prefix $p$ using floating-point arithmetic with safe comparisons instead of the current base-10 prefix would allow PUnits to support Imperial units more easily. PUnits performance in annotation mode also warrants further investigation. We remain optimistic in this regard: SMT solver performance has been steadily improving, we can explore different constraint encodings, and we can employ alternative solvers.

We plan to extend support for a more expressive form of parametric polymorphism, enabling methods such as myDivide $(x, y)$, which returns a unit computed as a function of the method arguments' units. Relationships between units could be explicitly declared via declaration annotations or automatically inferred from the typing constraints in the method body.

Units of measurements are important in many software domains. PUnits builds on techniques from type qualifier systems and constraint-based type inference, expanding them to the first system that provides a practical unit type system. Our implementation and evaluation of PUnits shows that these techniques are necessary and are effective in ensuring the unit correctness of real-world programs. PUnits provides an expressive and precise analysis with strong static guarantees. The system is easy to use and can make unit-correct code a reality. Our approach and implementation can be used to define other pluggable type systems. One definition of the type rules can be used to provide all three usage modes.

\section{ACKNOWLEDGMENTS}

We thank the reviewers for their valuable feedback on this paper. We acknowledge the support of the Natural Sciences and Engineering Research Council of Canada (NSERC) Discovery Grants program, RGPIN-05799-2014 and RGPIN-2020-05502, and an Early Researcher Award from the Government of Ontario. This work was also supported by AFRL contract FA8750-15-C-0010. The views and conclusions contained herein are those of the authors and should not be interpreted as necessarily representing the official policies or endorsements, either expressed or implied, of AFRL, DARPA, NSERC, or the Governments of Ontario, Canada, or the United States.

\section{REFERENCES}

Eric Allen, David Chase, Victor Luchangco, Jan-Willem Maessen, and Guy L Steele Jr. 2004. Object-oriented units of measurement. Object-Oriented Programming, Systems, Languages \& Applications (OOPSLA) 39, 10 (2004), $384-403$.

Oscar Bennich-Björkman and Steve McKeever. 2018. The next 700 unit of measurement checkers. In Software Language Engineering (SLE). 121-132.

Tom Benson. 2014. Reynolds Number. https://www.grc.nasa.gov/WWW/BGH/reynolds.html

Mars Climate Orbiter Mishap Investigation Board. 1999. Mars climate orbiter mishap investigation board: Phase I report. Jet Propulsion Laboratory.

Gilad Bracha. 2004. Pluggable type systems. In OOPSLA workshop on revival of dynamic languages, Vol. 4.

Travis Carlson and Eric Van Wyk. 2017. Type qualifiers as composable language extensions. In Generative Programming: Concepts \& Experiences (GPCE). ACM, 91-103.

Charles Zhuo Chen and Werner Dietl. 2018. Don't Miss the End: Preventing Unsafe End-of-File Comparisons. In NASA Formal Methods Symposium. Springer, 87-94.

The Coq Development Team. 2004. The Coq Proof Assistant. https://coq.inria.fr 
Daikon. 2003. Fix bug that printed milliseconds, not seconds. https://github.com/codespecs/daikon/commit/ a421b229bbed7da94bd3636c0eb03b716d151d5d

Daikon. 2004. Multiply dkconfig_compile_timeout by 1000 to convert from seconds to milliseconds. https://github.com/ codespecs/daikon/commit/1c74cbe4e1fe74d2ef2fc30c577482786d0aca5c

Jean-Marie Dautelle and Werner Keil. 2010. JSR 275: Units Specification API. https://jcp.org/en/jsr/detail?id=275

Jean-Marie Dautelle, Werner Keil, and Leonardo Lima. 2016. JSR 363: Units of Measurement API. https://jcp.org/en/jsr/ detail?id=363

Leonardo De Moura and Nikolaj Bjørner. 2008. Z3: An efficient SMT solver. In Tools and Algorithms for the Construction and Analysis of Systems (TACAS). Springer, 337-340.

Werner Dietl, Stephanie Dietzel, Michael D Ernst, Kivanç Muşlu, and Todd W Schiller. 2011a. Building and using pluggable type-checkers. In International Conference on Software Engineering (ICSE). ACM, 681-690.

Werner Dietl, Sophia Drossopoulou, and Peter Müller. 2007. Generic Universe Types. In European Conference on ObjectOriented Programming (ECOOP). Springer, 28-53.

Werner Dietl, Michael D Ernst, and Peter Müller. 2011b. Tunable Static Inference for Generic Universe Types. In European Conference on Object-Oriented Programming (ECOOP). Springer, 333-357.

Michael D Ernst, Alex Buckley, Werner Dietl, Doug Lea, Srikanth Sankaran, and Oracle. 2012. JSR 308: Annotations on Java Types. https://jcp.org/en/jsr/detail?id=308

Michael D Ernst, Jeff H Perkins, Philip J Guo, Stephen McCamant, Carlos Pacheco, Matthew S Tschantz, and Chen Xiao. 2007. The Daikon system for dynamic detection of likely invariants. Science of computer programming 69, 1-3 (2007), $35-45$.

Narain H. Gehani. 1977. Units of measure as a data attribute. Computer Languages 2, 3 (1977), 93-111.

Narain H. Gehani. 1985. Ada's derived types and units of measure. Software: Practice and Experience 15, 6 (1985), 555-569.

Sudheendra Hangal and Monica S Lam. 2009. Automatic dimension inference and checking for object-oriented programs. In International Conference on Software Engineering (ICSE). IEEE Computer Society, 155-165.

Mark Hills, Feng Chen, and Grigore Roşu. 2012. A rewriting logic approach to static checking of units of measurement in C. Electronic Notes in Theoretical Computer Science 290 (2012), 51-67.

Lingxiao Jiang and Zhendong Su. 2006. Osprey: a practical type system for validating dimensional unit correctness of C programs. In International Conference on Software Engineering (ICSE). ACM, 262-271.

Wesley Johnston. 2012. Close by swipe velocity checks are wrong. https://bugzilla.mozilla.org/show_bug.cgi?id=765069

Nahid Juma, Werner Dietl, and Mahesh Tripunitara. 2020. A computational complexity analysis of tunable type inference for Generic Universe Types. Theoretical Computer Science 814 (2020), 189-209.

Ted Kaminski, Lucas Kramer, Travis Carlson, and Eric Van Wyk. 2017. Reliable and automatic composition of language extensions to C: the ableC extensible language framework. Proceedings of the ACM on Programming Languages 1, OOPSLA (2017), 98.

Andrew Kennedy. 2009. Types for units-of-measure: Theory and practice. In Central European Functional Programming School. Springer, 268-305.

Andrew J Kennedy. 1997. Relational parametricity and units of measure. In Principles of Programming Languages (POPL). ACM, 442-455.

Sebastian Krings and Michael Leuschel. 2013. Inferring physical units in B models. In Software Engineering and Formal Methods (SEFM). Springer, 137-151.

Andreas Gullberg Larsen. 2018. Units.NET. https://github.com/angularsen/UnitsNet

Jens Melzer. 2015. Autoboxing Performance. https://effective-java.com/2015/01/autoboxing-performance

Dominic Orchard, Andrew Rice, and Oleg Oshmyan. 2015. Evolving Fortran types with inferred units-of-measure. fournal of Computational Science 9 (2015), 156-162.

Sam Owre, Indranil Saha, and Natarajan Shankar. 2012. Automatic dimensional analysis of cyber-physical systems. In Formal Methods. Springer, 356-371.

Matthew M Papi, Mahmood Ali, Telmo Luis Correa Jr, Jeff H Perkins, and Michael D Ernst. 2008. Practical pluggable types for Java. In International Symposium on Software Testing and Analysis (ISSTA). ACM, 201-212.

Matthias C. Schabel and Steven Watanabe. 2010. Boost.Units. http://www.boost.org/doc/libs/1_65_1/doc/html/boost_units. html

Scott Wlaschin. 2012. Units of measure - Type safety for numerics. https://fsharpforfunandprofit.com/posts/units-of-measure

Andrew K Wright and Matthias Felleisen. 1994. A syntactic approach to type soundness. Information and Computation 115, 1 (1994), 38-94. 